\title{
Ethische Richtlinien für die positiv-psychologische Praxis
}

\section{Aaron Jarden · Tayyab Rashid • Annalise Roache - Tim Lomas · Johannes Heekerens •} Aljoscha Dreisörner

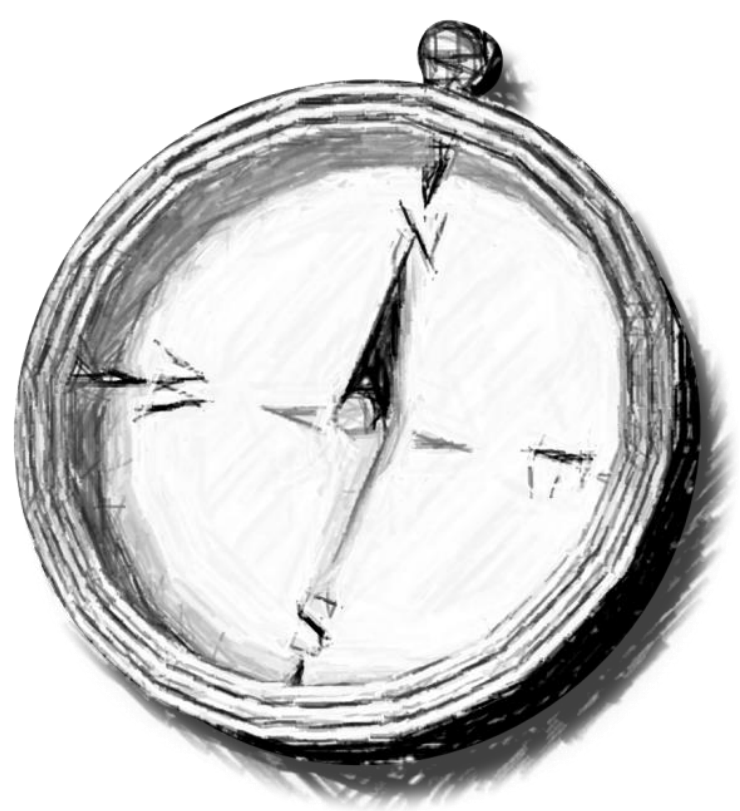




\section{Inhaltsverzeichnis}

Vorbemerkungen................................................................ 4

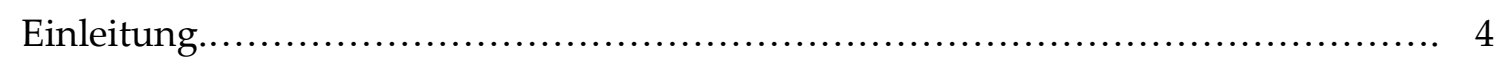

Berufsverbände und ethische Richtlinien für die positive-psychologische Praxis...... 4

Ziele der Richtlinien............................................................. 5

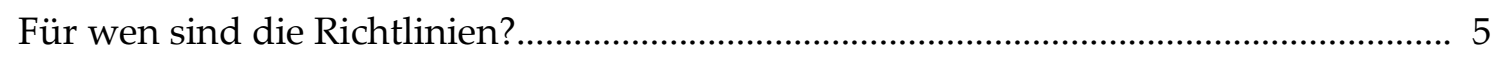

Vorläufiger Charakter der Richtlinien........................................... 5

Zentrale Begriffe............................................................... 6

Abschnitt Eins: Werte, Stärken, and Prinzipien...................................... 8

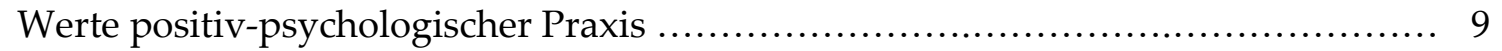

Ethische Prinzipien positiv-psychologischer Praxis .............................. 10

Abschnitt Zwei: Anwendungen der ethischen Richtlinien in der Praxis.................. 13

Drei wichtige Überlegungen.................................................. 13

Ethische Entscheidungsfindung............................................. 13

Überlegungen zur beraterischen Arbeit.......................................... 14

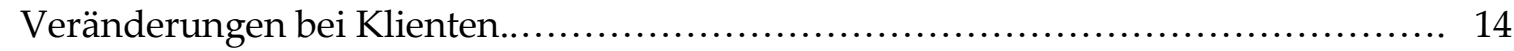

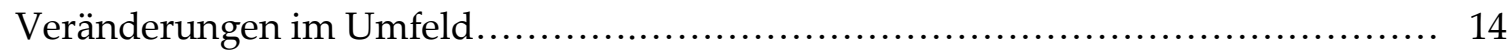

Aktuelle emotionale Verfassung und Wohlbefinden............................... 15

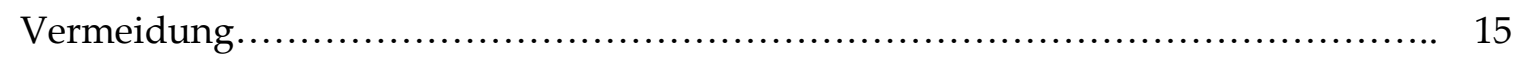

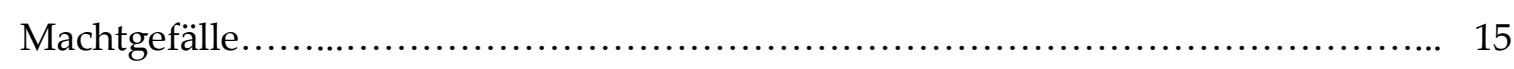

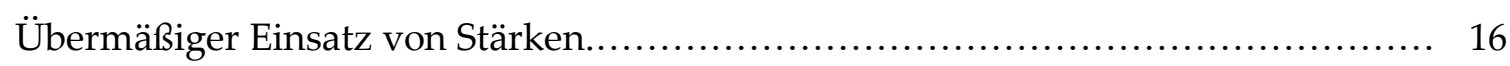

Praktische Weisheit............................................................. 16

Resilient bleiben und Burnout verhindern........................................ 16

Nachweis des Ausbildungshintergrunds und der fachlichen Kompetenz.............. 16

Würdigung des kulturellen Kontextes ........................................ 17

Ethische Dilemmata lösen - Eine Schritt für Schritt Anleitung.......................... 18

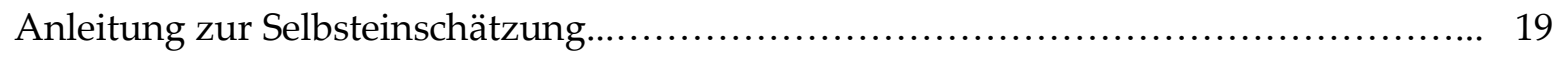

Abschnitt Drei: Fallbeispiele ethischer Praxis...................................... 20

Fallbeispiel 1: Kultur - kultursensible Anwendung von PPI....................... 20

Fallbeispiel 2: Coaching - Coachees in sich verändernden Umständen.............. 20

Fallbeispiel 3: Klinik - Arbeit mit Traumata....................................... 21

Fallbeispiel 4: Unternehmen - Druck Daten falsch auszulegen...................... 22 
Fallbeispiel 5: Mentoring - Motivation „unwilliger” Teilnehmer in einer ungleichen

Beziehung.

Fallbeispiel 6: Bildung - Durchführung von Programmen zur Steigerung von

Wohlbefinden wenn bestehende Praktiken dieser Absicht entgegenstehen

Relevante Richtlinien............................................................ 26

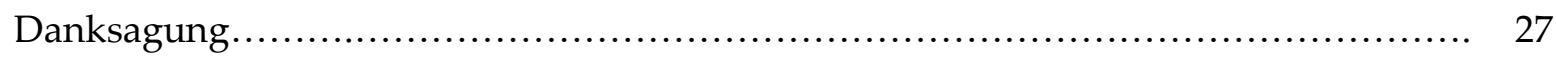

Erste Unterzeichner der ethischen Richtlinien für die positiv-psychologische Praxis...... 28

Literaturverzeichnis............................................................. 29

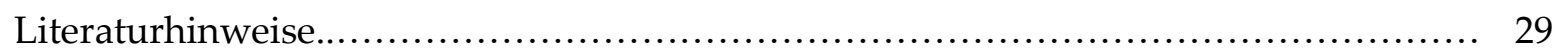

Erklärung: Diese Richtlinien sind das Ergebnis einer unabhängigen Arbeitsgruppe, die von Aaron Jarden, Tayyab Rashid, Annalise Roache und Tim Lomas geleitet wurde. Die deutsche Übersetzung stammt von Johannes Heekerens und Aljoscha Dreisörner. Die Richtlinien sind nicht innerhalb einer Organisation oder eines Berufsverbandes entstanden und von diesen unabhängig. Allerdings wurden unterschiedliche Akteure in die Entwicklung und Verbesserung dieser ersten Version einbezogen. Die Autoren haben die Absicht, die Richtlinien alle zwei Jahre zu aktualisieren, um deren Tiefe und Anwendungsumfang zu verbessern. Feedback nimmt Aaron Jarden (aaron.jarden@unimelb.edu.au) gerne entgegen. 


\section{Vorbemerkungen}

\section{Einleitung}

Ethische Perfektion ist wahrscheinlich ein utopisches Ideal, auch deshalb, weil Menschen, inklusive der Praktiker Positiver Psychologie (PPP), fehlbar, verletzlich und unvollkommen sind. Die Komplexität gegenwärtigen Lebens, das geprägt ist von digitalen Welten, unberechenbaren Klimaveränderungen, Flüchtlingskrisen, sich entfaltender Identitäten und zunehmender wirtschaftlicher Polarisierung, verschärft menschliche Vulnerabilitäten.

Psychologische Dienstleistungen, insbesondere solche deren explizites Ziel die Wiederherstellung oder Förderung von Wohlbefinden ist, werden nicht in der Abwesenheit konkurrierender Interessen und ethischer Dilemmata angeboten. Keine einzelne Zusammenstellung von ethischen Richtlinien, Standards, oder gar Statuten kann dem Spektrum menschlicher Komplexität vollständig gerecht werden. Eine Zusammenstellung breit abgestützter Werte, persönlicher Stärken und Prinzipen kann uns trotz allem Orientierung bieten, wie wir ethischere Entscheidungen treffen können, insbesondere wenn wir positivpsychologischer Interventionen (PPI) anwenden, deren allgemeines Ziel die Förderung von Wohlbefinden ist.

Diese Richtlinien enthalten a) Werte, b) Stärken und c) Prinzipen für die positiv-psychologische Praxis. Es ist wichtig, sich in Erinnerung zu rufen, dass Richtlinien üblicherweise in weitere Regelwerte eingebettet sind, die sich je nach Situation und Rechtsprechung unterscheiden können. Beispielsweise könnten Richtlinien den untersten Teil einer präskriptiven Hierarchie bilden (siehe Abbildung 1):

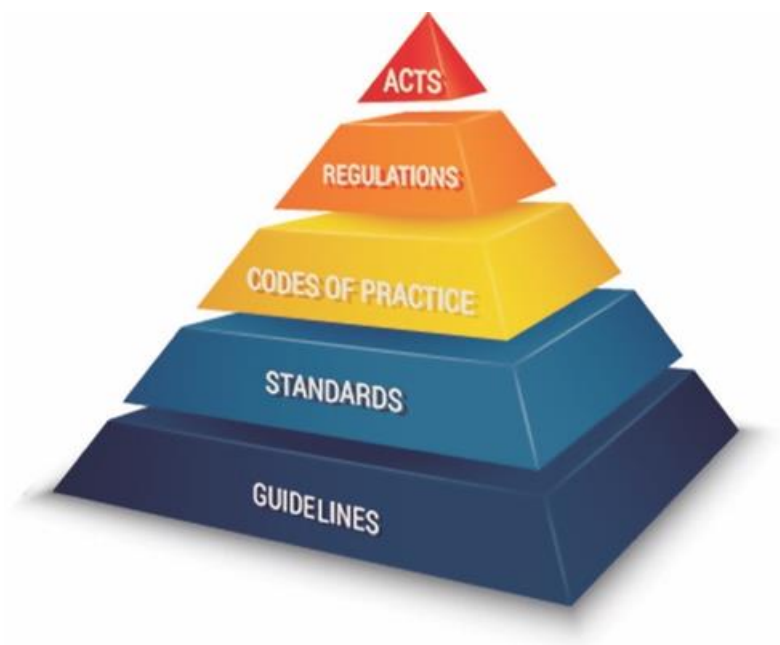

Abbildung 1. Richtlinien und weitere Regelwerke.

Deshalb ist es wichtig, die Richtlinien zusammen mit anderen Regelwerken zu betrachten. Einzelpersonen oder Organisationen mögen sich auf diese Richtlinien für die „positivpsychologische Praxis“ beziehen und sie befürworten, jedoch kann es laut der Rechtsprechungen mancher Länder illegal sein, sich als „Positiver Psychologe“ zu bezeichnen bitte seien Sie sich der für sie geltenden lokalen Beschränkungen und Vorgaben im Klaren. ${ }^{1}$

\footnotetext{
${ }^{1}$ Anmerkung des Übersetzers: In Deutschland ist „Psychologe“ eine geschützte Berufsbezeichnung, die ein abgeschlossenes Diplom oder einen Masterabschluss in Psychologie erfordert. Von einem „Positiven Psychologen“ kann erwartet werden, dass es sich um einen Psychologen mit Spezialisierung im Bereich Positive Psychologie handelt. Ob ein Titelschutz für "Positive Psychologen" besteht, kann vom Einzelfall abhängen und juristische Entscheidungen bleiben abzuwarten.
} 
Berufsverbände und ethische Richtlinien für die positive-psychologische Praxis

Wenn ein Praktiker Mitglied eines relevanten Berufsverbandes ist (z.B., Amerikanische Psychologische Gesellschaft, Deutsche Gesellschaft für Psychologie, International Coach Federation) hat er zunächst und vor allem deren Standards, deren Kodex oder deren Richtlinien für das berufspraktische Handeln heranzuziehen. Wir erwarten, dass Praktiker, die durch ein Genehmigungs- oder Qualifikationsgremiums (z.B., dem College of Psychologists) legitimiert sind, über ausreichend Ausbildung, Training und Erfahrung in der psychologischen Praxis oder einem angrenzenden Berufsfeld verfügen, sowie dass sie mit den Regeln,

Standards, Regularien, Statuten und Abläufen psychologischer Praxis entsprechend der für sie geltenden Rechtsprechung vertraut sind.

Die hier vorgestellten ethischen Richtlinien für die positiv-psychologische Praxis sind entwickelt worden, um existierende Rechts- und Berufsordnungen und Richtlinien zu ergänzen, nicht zu ersetzen. Als solche stellen diese Richtlinie auch eine erste Orientierung für Praktiker dar, die keinem Berufsverband angehören.

\section{Ziele der Richtlinien}

Warum braucht es eine Zusammenstellung ethischer Richtlinien? Ziel dieser Richtlinien ist, die ethische Praxis durch eine Verpflichtung zu wissenschaftlich informierten und evidenzbasierten Verfahren aus der Positiven Psychologie (PP) zu verbessern. Ihre Absicht ist, dafür zu werben, eine (angemessene) Treue zur Wissenschaft des Wohlbefindens beizubehalten und insbesondere Praktiker dafür zu sensibilisieren, diese Treue bei der Selbstdarstellung und dem Marketing möglicher Vorteile zu berücksichtigen. Die Richtlinien versuchen bei PPP und anderen ein klareres Verständnis darüber zu befördern, was ethisch informierte Praxis in der Positiven Psychologie bedeutet. Mit Unterstützung solcher Richtlinien wird von PPP erwartet, sich zu Folgendem zu bekennen:2

a) sich genau mit der Forschung auseinanderzusetzen und die eigene Praxis auf eine Wissenschaftsbasis zu stellen;

b) ihr Wissen durch fortlaufendes Lernen und berufliche Weiterbildung kontinuierlich weiterzuentwickeln;

c) sorgfältig und genau die Grenzen des aktuellen Wissens in Bezug auf PPI zu vermitteln, ebenso wie die möglichen Vorteile und Fallstricke spezifischer PPI und Programme zur Steigerung des Wohlbefindens; und

d) das Wohlbefinden von Klienten, sowie positive und negative Veränderungen im Wohlbefinden während der Leistungserbringung zu überwachen.

Für wen sind diese Richtlinien?

Der Zweck dieser Richtlinien ist, optimales ethisches Verhalten von PPP zu ermöglichen und dadurch anderen Menschen Gutes zu tun und sie vor möglichem Schaden zu bewahren. Als solche sind diese Richtlinien für Experten (z.B., Psychologen) und andere (z.B., Studierende oder solche ohne Berufszugehörigkeit) gedacht, die PPI einsetzen oder Wissen aus dem Bereich der Positiven Psychologie in Ihrer Arbeit zur Anwendung bringen. Diese Richtlinien sind keine Anleitung für die Anwendung bestimmter PPI.

\footnotetext{
${ }^{2}$ Wir haben vier Beispiele zur Veranschaulichung ausgewählt. Die Liste könnte erweitert warden.
} 


\section{Vorläufiger Charakter der Richtlinien}

Wir weisen darauf hin, dass die aktuellen Richtlinien (Version 1.0, 19. Juli 2019) vorläufig sind und alle zwei Jahre durch einen sich wiederholenden beratenden Prozess mit wichtigen Interessensvertretern überarbeitet werden. Diese Regelmäßigkeit der Überarbeitung der Richtlinien dient dazu, auf kontextabhängige Veränderungen reagieren zu können und sich stätig weiterentwickelnde lokale und globale Themen zu berücksichtigen. In diesem Sinne heißen wir Rückmeldungen zu den hier beschriebenen Richtlinien jederzeit willkommen.

Es ist wichtig zu verstehen, dass nur englischsprachige, zumeist westliche Länder die Entwicklung dieser Richtlinien beeinflusst haben und die Richtlinien entsprechend begrenzt sind. Weil dies ein lebendiges und wachsendes Dokument ist, das durch eine Versionsnummer identifiziert ist, bleibt allerdings für zukünftige Versionen zu hoffen, dass Einflüsse von Kodizes und Richtlinien aus nicht-englischsprachigen und nicht-westlichen Ländern aufgenommen werden.

\section{Zentrale Begriffe}

\section{$\underline{\text { Klient }}$}

Praktiker arbeiten mit Klienten. „Klient“ ist eine allgemeine Bezeichnung für die Empfänger jeglicher Dienstleistungen, zu denen (jedoch nicht ausschließlich) Einzelpersonen, Paare, Familien, Gruppen, Organisationen, Sponsoren, oder andere bestimmbare soziale Einheiten gehören.

\section{Kodizes vs. Richtlinien}

Ein Kodex hat üblicherweise rechtliche oder andere vollstreckbare Auswirkungen, wohingegen Richtlinien Best Practices empfehlen, die für gewöhnlich nicht reguliert oder überwacht werden. Richtlinien unterstellen ihrem Wesen nach, dass Situationen sich unterscheiden und komplex sind und dass das Befolgen der Richtlinien oftmals voraussetzt, dass Praktiker praktische Weisheit anwenden - eine Kombination aus Erfahrung und gesundem Menschenverstand.

$\underline{\mathrm{PP}}$

PP: Positive Psychologie. Es gibt viele unterschiedliche Konzeptualisierungen und Definitionen für PP, aber die meisten verstehen sie als die wissenschaftliche Erforschung und Förderung von übergreifenden Konstrukten wie "Glück", „Wohlbefinden“ und „persönlicher Entwicklung". Beispielsweise definieren Lomas, Hefferon und Ivtzan (2015, S. 1347) PP als "the science and practice of improving wellbeing." ${ }^{3}$ In diesem Zusammenhang gibt es auch den Begriff der "angewandten Positiven Psychologie“, welcher einfach als die Anwendung der PP in realen Situationen zu verstehen ist, welche PPI einschließt.

\section{$\underline{\text { PPI }}$}

PPI: Positiv-psychologische Interventionen. In der Literatur wird eine Trennung vorgenommen zwischen „Positive Psychologie Interventionen“ und „positiv-psychologischen Interventionen“ - für eine Überblick und Erläuterung siehe Hone, Jarden und Schofield (2015). Hier berücksichtigen wir den breiter gefassten Begriff positiv-psychologischen Interventionen, der alle Aktivitäten aus vielen Disziplinen (z.B. aus anderen Teilgebieten der Psychologie zusätzlich zur Positiven Psychologie) umfasst, deren Ziel die Steigerung psychologischen

\footnotetext{
${ }^{3}$ Übersetzt in etwa ,die Wissenschaft und Praxis zur Förderung von Wohlbefinden“.
} 
Wohlbefindens ist. Eine verbreitete Definition für positiv-psychologische Interventionen ist: “A positive psychological intervention promotes positive emotions, behaviours, and/or thoughts, thereby increasing the wellbeing of an individual or group" 4 (Schueller, Kashdan, \& Parks, 2014, p. 92).

\section{$\underline{P P P}$}

PPP: Praktiker der Positiven Psychologie. Ein Praktiker, der einschlägig ausgebildet ist (z.B. durch Psychotherapie, Beratung oder Coaching) und ein spezifisches PP Training bei einem seriösen Weiterbildungsanbieter absolviert hat, welches ein Verständnis des wissenschaftlichen Forschungsprozesses und seiner Anwendungen in der Bereitstellung von angewandter Positiver Psychologie (z.B., PPI) ermöglicht. ${ }^{5}$

\section{Stärken}

Allgemein gesprochen machen sich Richtlinien und Kodizes für bestimmte

Persönlichkeitsmerkmale stark, die Vordenker des jeweiligen Felds willkürlich ausgewählt haben. Diesen Richtlinien legten wir die Character Strengths and Virtues (CSV: Peterson \& Seligman, 2004) zugrunde, ein empirisch validiertes und kulturübergreifend bestätigtes Modell von Charakterstärken (McGrath, 2014). Die CSV definieren Charakterstärken als universelle Persönlichkeitseigenschaften, die für sich genommen wertvoll sind und nicht notwendigerweise instrumentellen Zielen dienen. Vielmehr handelt es sich um verhaltenswirksame Persönlichkeitseigenschaften, die eine Person darin unterstützen und dazu anleiten, ihr Verhalten in verantwortungsvoller und ethischer Art und Weise im Sinne des eigenen Wohlergehens und des Wohlergehens anderer auszurichten. Charakterstärken drücken, vereinfacht gesagt, aus, wer eine Person ist (bzw. deren Charakter) und Werte drücken aus, was sich eine Person verpflichtet fühlt (bzw. deren Prinzipien und Handlungen).

\section{$\underline{\text { Praktiker }}$}

Der Ausdruck „Praktiker” wird allgemein verwendet für eine Person, die Verantwortung für die Bereitstellung einer der PP verwandten Dienstleistung trägt. „Praktiker“ beinhaltet jeden, der eine Rolle oder Rollen wie Berater, Psychotherapeut, psychosoziale Fachkraft, Coach, Trainer, Mentor oder Erzieher innehat, ist allerdings nicht auf diese beschränkt. ${ }^{6}$

\section{Prinzipien}

Prinzipien richten Aufmerksamkeit auf wichtige ethische Verantwortlichkeiten aus. Handlungen foglen auf Basis bestimmter Prinzipien.

\footnotetext{
${ }^{4}$ Übersetzt in etwa „Eine positiv-psychologische Intervention fördert positive Emotionen, Verhaltensweisen, und/oder Gedanken, wodurch das Wohlbefinden einer Einzelperson oder einer Gruppe erhöht wird.“

5 Wer kann sich als PPP bezeichnen? Zurzeit gibt es keine klare Grenze in Bezug auf das notwendige Ausmaß, Trainingsniveau oder Qualifikationslevel. Beispielsweise könnte sich jemand nach einem zweitägigen Kurs, einer sechsmonatigen akkreditierten Zertifizierung oder einem zweijährigen Masterprogramm in Angewandter Positiver Psychologie als PPP bezeichnen? Außerdem sind supervidierte Praxis (z.B., Peer- oder Einzelsupervision) und Onlineberatung zu empfehlen. Weitere Erläuterungen von den einschlägigen Berufsverbänden zu angemessenem Training und Supervision sind notwendig (und tatsächlich werden diese Themen bereits von relevanten Akteuren berücksichtig und angegangen).

${ }^{6}$ Der Ethos und die Prinzipien, die in diesen Richtlinien enthalten sind, könnten gleichermaßen auf Wohlbefindensforscher angewandt werden, weil Forscher in dem Sinne als Praktiker verstanden werden können, als dass sie mit Teilnehmern arbeiten, die ähnlich wie Klienten betrachtet werden können.
} 


\section{Werte}

Werte sind Glaubenssätze in Bezug auf erwünschte Ergebnisse, die von Einzelpersonen verinnerlicht und durch Gruppen geteilt werden (bzw., weitgefasste Anschauungen über die Welt und wie sie sein sollte). Werte sind situationsübergreifend, beeinflussen welche Handlungen wir wählen und wie wir andere und uns selbst bewerten; sie sind nach ihrer relativen Wichtigkeit geordnet (Schwartz, 2006). Werte können ein wichtiger Ausdruck einer allgemeinen ethischen Verpflichtung sein, die, wenn sie als Prinzip formuliert wird, eine genauere Definition und Handlungsorientierung erhält. In anderen Worten, Werte informieren Prinzipien. In diesem Sinne sind die Werte, welche in diesen Richtlinien zum Ausdruck kommen, jene, welche die Autoren als allgemein in der Gemeinschaft der PP geteilt ansehen. 


\section{Abschnitt Eins: Werte, Stärken und Prinzipien}

Die ethischen Richtlinien für die positiv-psychologische Praxis beinhalten die folgenden Werte, Stärken und Prinzipien. Sowohl Werte als auch Stärken unterstützen die Umsetzung ethischer Prinzipien, wie in Tabelle 1 dargestellt.

Tabelle 1

Werte, Stärken und Prinzipien der positiv-psychologischen Praxis

Werte $^{7} \quad$ Stärken ${ }^{8} \quad$ Prinzipien
1. Den Schutz von Klienten und
1. Ehrlichkeit.
1. Wohltätigkeit/Schadensvermeidung. anderen gewährleisten.
$\begin{array}{lll}\text { 2. Persönliche Not und Leiden } & \text { 2. Fairness. } & \text { 2. Verantwortungsvolle Fürsorge. }\end{array}$ lindern.
3. Die Integrität der Praktiker- Klient Beziehung sicherstellen.
3. Soziale Intelligenz.
3. Achtung der Rechte und Würde des Menschen.
4. Die Unterschiedlichkeit menschlicher Erfahrungen und Kulturen würdigen.

4. Teamwork.

4. Vertrauenswürdigkeit.
5. Förderung eines
Selbstverständnisses, das bedeutungsvoll für die betreffende(n) Person(en) ist.

5. Freundlichkeit.

5. Gerechtigkeit.
6. Die Qualität professionellen
6. Vorsicht.
6. Autonomie. Wissens und seiner Anwendung erhöhen.
7. Die Beziehungsqualität
7. Weitsicht. zwischen Menschen erhöhen.
8. Persönliche Wirksamkeit erhöhen.
9. Sich einsetzen für die faire und adäquate Bereitstellung von Beratung, Psychotherapie und Coaching.

\section{Urteilsvermögen.}
9. Selbstkontrolle.

10. Ausdauer.

11. Mut.

\footnotetext{
${ }^{7}$ Unsere Werte wurden auf Basis ähnlicher Richtlinien ausgewählt und aus diesem Grund können viele der Werte unterschiedlichen Kategorien zugeordnet werden. Beispielsweise könnte Gerechtigkeit als Wert oder als Prinzip verstanden werden. Vertrauenswürdigkeit könnte als Stärke (Ehrlichkeit), als Wert oder als Prinzip verstanden werden. Viele dieser Konstrukte wurden in diesem Sinne diskutiert. Die Werte wurden außerdem von den Autoren dieser Richtlinien gemäß ihrer relativen Wichtigkeit geordnet.

${ }^{8}$ Stärken 1- 7 sind danach geordnet, wie häufig sie in 10 ähnlichen Ethikkodizes (z.B., dem ethischen Rahmenwerk der Britischen Gesellschaft für Beratung und Psychotherapie) genannt werden. Stärken 8-11 wurden hinzugefügt, weil die Autoren sie für wichtig erachteten, um die sechs Prinzipien zu wahren.
} 
Außerdem:

- Fast alle Kodizes legen eine Reihe von Persönlichkeitsmerkmalen und persönlichen Qualitäten fest, wohingegen diese Richtlinien auf Stärken und beobachtbares Verhalten fokussieren.

- Die Listen der Werte, Stärken und Prinzipien sind in keiner Weise erschöpfend, sondern vielmehr ein erster Versuch zu formulieren, was zurzeit als am zweckdienlichsten für die Praxis erachtet wird. Wenn diese Richtlinien iterativ über die Zeit angepasst werden, begleitet von einer entsprechenden Debatte innerhalb der Gemeinschaft der $\mathrm{PP}$, ist anzunehmen, dass sich die Bestand teile ebenfalls weiterentwickeln.

- Die Listen der Werte, Stärken und Prinzipien ist außerdem nicht bindend.

- Ein Stärkenmodell (das Values in Action ${ }^{9}$ Rahmenmodell) wurde ausgewählt, weil es das in der Literatur am meisten verbreitetste war und auf solider Wissenschaft beruht.

Werte von positiv-psychologischen Praktikern

Die grundlegenden Werte von PPP beinhalten eine Verpflichtung zu:

- Den Schutz von Klienten und anderen gewährleisten. Dies beinhaltet eine stetige und wachsame Aufmerksamkeit in Bezug darauf, wie ein Klient auf die eigenen Dienstleistungen reagiert. Außerdem bedeutet es, die Grenzen der eigenen Kapazität und Expertise zu kennen (und in diesem Sinne zu erkennen, wann ein Klient professionelle Hilfe aus anderen Bereichen benötigt oder ob eine Gefahr für die Öffentlichkeit besteht).

- Persönliche Not und Leiden lindern (falls in den Grenzen der eigenen Expertise). Damit ist gemeint, PPI in situativ relevanter Art und Weise anzuwenden, um Leiden von Einzelpersonen zu lindern, die Dienstleistungen in verschiedenen professionellen Kontexten (z.B. Therapie, Coaching, Gesundheit) in Anspruch nehmen.

- Die Integrität der Praktiker-Klient Beziehung sicherstellen. Dies beinhaltet Respekt vor persönlichen Grenzen und eine Verpflichtung zu professionellen Praktiken wie Datenschutz und Vertraulichkeit.

- Die Unterschiedlichkeit menschlicher Erfahrungen und Kulturen würdigen. Damit ist gemeint, in der Arbeit mit Klienten nicht nach dem Gießkannenprinzip oder „one-size-fitsall“ vorzugehen, sondern die Unterschiedlichkeit der einzigartigen Entwicklungswege von Menschen, sowie kontextuelle und kulturelle Hintergründe, zu respektieren und diese Unterschiede sogar zu zelebrieren.

- Förderung eines Selbstverständnisses, das bedeutungsvoll für die betreffende(n) Person(en) ist. Damit ist gemeint, dass der Einzelne Ziele entwickelt und verfolgt, die nicht nur für ihn selbst erfüllend sind, sondern auch zum Gemeinwohl beitragen.

- Die Qualität professionellen Wissens und seiner Anwendung erhöhen. Damit ist gemeint, das individuell Möglichste zu tun, um Entwicklungen in der Literatur Rechnung zu tragen und über Fortschritte auf dem Laufenden zu bleiben.

- Die Beziehungsqualität zwischen Menschen erhöhen. Damit ist gemeint, anzuerkennen, dass Menschen unvermeidlich in sozialen Beziehungen und Netzwerken eingebunden sind, sowie danach zu streben, diese Bindungen so weit wie möglich zu verbessern.

- Persönliche Wirksamkeit erhöhen. Damit ist gemeint, danach zu streben, Klienten dabei zu unterstützen, effektiver in der Welt und in den einzigartigen Situationen, in denen sie sich wiederfinden, zu handeln.

\footnotetext{
${ }^{9}$ Anm. d. Ü.: Wörtlich übersetzt „Werte in Aktion“. Gemeint ist, dass die behandelten Stärken Werten zugeordnet sind, die wiederum in Handlungen zum Ausdruck kommen.
} 
- Sich einsetzen für die faire und adäquate Bereitstellung von Beratung, Psychotherapie und Coaching. Dies könnte das Einstehen für Dienstleistungen für schwer zu erreichenden Populationen beinhalten, oder, falls möglich, eine Preisstruktur anzubieten, die auch Menschen mit weniger Mitteln ermöglicht von Leistungen zu profitieren.

\section{Stärken positiv-psychologischer Praktiker}

Die persönlichen Stärken der Praktiker sind wichtig, weil sie einen Einfluss auf die PraktikerKlient Beziehung haben. Viele der Stärken, die als wichtig für die Bereitstellung positivpsychologischer Leistungen erachtet werden sind gute persönliche Qualitäten. Es ist unangemessen vorzuschreiben, dass alle Praktiker diese Stärken besitzen, weil es grundlegend ist, dass diese persönlichen Stärken tief in der betreffenden Person verankert und aus persönlichem Einsatz heraus entwickelt werden und nicht etwa, weil dies den Anforderungen einer externen Autorität entspricht. Eine thematische Analyse der Prinzipien und Werte, die von wichtigen Richtlinien (siehe Relevante Richtlinien) unterstützt werden, zeigte, dass von den folgenden Charakterstärken angenommen werden kann, dass sie ethisches Verhalten und ethischer Entscheidungsfindung begünstigen:

- Ehrlichkeit: Die Wahrheit sagen; sich selbst in aufrechter Art und Weise darstellen, sowie in ehrlicher Absicht handeln; auf Täuschung verzichten; Verantwortung für die eigenen Gefühle und Handlungen übernehmen.

- Fairness: Alle Menschen im Geiste von Fairness und Gerechtigkeit gleich zu behandeln; eigene Gefühle nicht Entscheidungen über andere verzerren lassen; jedem eine faire Chance geben.

- Soziale Intelligenz: Sich der Motive und Gefühle anderer Personen bewusst sein; wissen, was in verschiedenen sozialen Situationen zu tun ist: wissen, was zu tun ist, um andere zu beruhigen.

- Teamwork: Gut als Mitglied einer Gruppe oder eines Teams arbeiten; der Gruppe loyal sein; seinen Anteil übernehmen.

- Freundlichkeit: Anderen Gefallen und Wohltaten tun; ihnen helfen; sie versorgen.

- Vorsicht: Entscheidungen, die einen selbst betreffen mit Sorgfalt treffen; keine unnötigen Risiken eingehen; nichts sagen oder tun, was man später bereuen könnte.

- Weitsicht: In der Lage sein, anderen weisen Rat zu erteilen; mit Sichtweisen auf die Welt ausgestattet sein, die für einen selbst und andere Sinn ergeben.

- Urteilsvermögen: Dinge durchdenken und sie von allen Seiten her betrachten; keine voreiligen Schlüsse ziehen; Entscheidungen auf eine solide Evidenzbasis stellen; die Fähigkeit haben, die eigene Meinung ändern zu können.

- Selbstkontrolle: Regulieren, wie man sich fühlt und was man tut; diszipliniert sein; eigene innere Impulse und Emotionen kontrollieren.

- Ausdauer: Hart arbeiten, um rechtzeitig zu beenden, was man angefangen hat, unabhängig vom Projekt; sich bei der Arbeit nicht ablenken lassen; Befriedigung aus erledigten Aufgaben ziehen.

- Mut: Eine mutige Person sein, die nicht vor Bedrohung, Herausforderung, Schwierigkeit oder Schmerz zurückschrickt; vertreten was richtig ist, selbst wenn es Widerstand gibt; den eigenen Überzeugungen entsprechend handeln.

Ethische Prinzipien für die positiv-psychologische Praxis

Jedes der sechs unten beschriebenen Prinzipien ist durch ein Beispiel guter Praxis ergänzt, das in Erwiderung auf das Prinzip entwickelt wurde. Ethische Entscheidungen, die stark von 
einem oder mehreren Prinzipien gestützt werden, ohne Widersprüche durch andere, können als starke moralische Intuitionen verstanden werden. Nichtsdestotrotz werden Praktiker vermutlich Umständen begegnen, in denen es unmöglich ist, alle anwendbaren Prinzipien in Einklang zu bringen und in denen eine Wahl zwischen den Prinzipien erforderlich sein kann. Eine Entscheidung oder eine Handlungsweise wird nicht notwendigerweise dadurch unethisch, dass sie umstritten ist oder weil andere Praktiker zu unterschiedlichen Schlüssen in derselben Situation gekommen wären. Die Verantwortung eines Praktikers ist es, alle relevanten Umstände so sorgfältig wie es vernünftigerweise erwartet werden kann in Betracht zu ziehen und für die getroffenen Entscheidungen in angemessen Art und Weise Verantwortung zu übernehmen.

\section{Wohltätigkeit/Schadensvermeidung}

Wohltätigkeit beinhaltet eine Verpflichtung zur Förderung des Wohlbefindens des Klienten und des Praktikers. Das Prinzip der Wohltätigkeit meint, basierend auf einer professionellen Beurteilung im besten Interesse des Klienten zu handeln. Es fokussiert darauf, strikt in den Grenzen der eigenen Kompetenz zu arbeiten und Dienstleistungen auf der Basis von adäquatem Training oder entsprechender Erfahrung anzubieten. Sicherzustellen, dass die besten Interessen des Klienten erfüllt werden, setzt eine systematische Kontrolle der eigenen Vorgehensweise und der Ergebnisse (z.B., Wohlbefinden) auf Basis der besten zur Verfügung stehenden Mittel voraus. Es wird als wichtig erachtet, dass wissenschaftliche Forschung und systematische Reflexion die eigene Praxis informieren. Je nach Rolle und Kontext gibt es üblicherweise eine Pflicht, Maßnahmen zu ergreifen, um die Qualität der angebotenen Leistungen zu verbessern und die eigenen Praxis durch kontinuierliche berufliche Weiterbildung auf den neuesten Stand zu bringen. Eine Pflicht, im besten Interesse eines Klienten zu handeln, wird dann besonders entscheidend, wenn die Arbeit mit Klienten stattfindet, deren Autonomie aufgrund von Alter, Unreife, fehlendem Verständnis, Bedrängnis, oder anderen bedeutsamen persönlichen Einschränkungen vermindert ist.

Schadensvermeidung, auf der anderen Seite, beinhaltet eine Verpflichtung Klienten vor Leid zu bewahren. Praktiker, die dieses Prinzip übernehmen:

- vermeiden sexuelle, finanzielle, emotionale oder irgendeine andere Form der Ausbeutung von Klienten;

- vermeiden Inkompetenz (z.B., angemessene Anwendung von PPI, bzw., wissen, was für wen wirkt) und Fehlverhalten;

- bieten keine Leistungen an, wenn sie aufgrund von Krankheit, persönlicher Umstände oder Intoxikation dazu nicht in der Lage sind.

Der Praktiker hat eine ethische Verantwortung, sich darum zu bemühen, jeglichen Schaden der einem Klienten zugefügt wurde, zu lindern, auch dann, wenn der Schaden unvermeidlich oder unbeabsichtigt war. Praktiker haben persönliche und berufliche Verpflichtungen, die Inkompetenz oder das Fehlverhalten anderer, wo angebracht, zur Sprache zu bringen. Sie sind auch verpflichtet, $z u$ jeglichen Untersuchungen und/oder Urteilsfindungen von einer in angemessener Art und Weise anerkannten Einrichtung oder Autorität beizutragen, die professionelles Verhalten betreffen, dass dem eines einigermaßen kompetenten Praktikers nicht entspricht und/oder das Risiko birgt, die Profession der PP zu diskreditieren. 


\section{Verantwortungsvolle Fürsorge}

Die positiv-psychologische Praxis fördert Wohlbefinden. In der Verfolgung dieses Ziels zeigen PPP, die dieses Prinzip übernehmen, ein aktives Interesse am Wohlergehen derer, mit denen sie arbeiten und erkennen die sozialen und institutionellen Kräfte an, die ihre Rolle als PPP regelt. Sie tragen zu allererst die Verantwortung dafür, das Wohlergehen derer, mit denen sie arbeiten, zu schützen. Sie erkennen an, dass Einzelpersonen, Familien, Gruppen oder Gemeinschaften in einer ungeschützten Lage sein können. Sie erkennen die Grenzen ihrer Expertise (inklusive der Abwesenheit von Wissen) an und verweisen auf andere Spezialisten, wann immer notwendig. Außerdem übernehmen sie Verantwortung und korrigieren jeglichen Schaden, der als Ergebnis einer ihrer Dienstleistungen entstanden ist. Von PPP wird auch erwartet, dass sie ein aktives und kontinuierliches Interesse an den empirischen und theoretischen Entwicklungen ihres Feldes zeigen, um sicherzustellen, dass ihr Wissen auf dem neuesten Stand der Evidenz in Bezug auf die PPI, die sie anwenden, bleibt.

Achtung der Rechte und Würde des Menschen

Im Einklang mit der Erklärung der Menschenrechte der Vereinten Nationen setzt der Respekt gegenüber den Rechten und der Würde des Menschen voraus, dass jede Person und alle Völker für sich genommen positiv bewertet werden, ihre Rechte anerkannt und respektiert werden und ihnen Respekt zuteil, sowie Würde als Bestandteil ihrer gemeinsamen Menschlichkeit gewährt wird. Praktiker, die dieses Prinzip umsetzen, zeigen Respekt, was eine Sensibilität gegenüber kultureller und sozialer Vielfalt voraussetzt. Dies beinhaltet die Anerkennung von Unterschieden zwischen Personen in Bezug auf ihre Kultur, Nationalität, Ethnie, Hautfarbe, Abstammung, Religion, Geschlecht, Beziehungsstatus, sexuelle Orientierung, physische oder geistige Fähigkeiten, Altern, sozio-ökonomischer Status, und/oder einem anderen persönlichen Merkmal, Zustand, oder Status. Solche Unterschiede sind ein integraler Teil von Personen und sollten als solche respektiert werden.

\section{Vertrauenswürdigkeit}

Vertrauenswürdig zu sein (Authentizität) bedeutet, das Vertrauen, welches dem Praktiker zu Teil wird, zu würdigen. Vertrauenswürdigkeit wird als Grundlage für das Verständnis und die Lösung ethischer Fragen betrachtet. Praktiker, die dieses Prinzip anwenden:

- handeln entsprechend dieses ihnen entgegengebrachten Vertrauens;

- bemühen sich, sicherzustellen, dass die bei Klienten geweckten Erwartungen mit angemessener Wahrscheinlichkeit erfüllt werden können;

- halten ihre Absprachen und Versprechen ein;

- betrachten Vertraulichkeit als eine Pflicht, die sich aus dem Vertrauen des Klienten ergibt;

- beschränken die Weitergabe vertraulicher Informationen, die Klienten betreffen, auf das Notwendigste, um den Zweck zu erfüllen, aufgrund dessen sie ursprünglich geteilt wurden - mit Ausnahme zwingender Offenlegung (z.B. durch ein Gesetz).

\section{Gerechtigkeit}

Gerechtigkeit beinhaltet die faire und unparteiische Behandlung aller Klienten und die Erbringung angemessener Leistungen. Das Prinzip der Gerechtigkeit verlangt, dass Praktiker, die dieses Prinzip anwenden, gerecht und fair gegenüber allen Kunden sind und deren Menschenrechte und Würde achten. Es lenkt die Aufmerksamkeit darauf, alle rechtlichen Anforderungen und Verpflichtungen gewissenhaft zu prüfen und auf mögliche Konflikte 
zwischen rechtlichen und ethischen Verpflichtungen zu achten. Gerechtigkeit bei der Verteilung von Leistungen erfordert die Fähigkeit, die Erbringung von Leistungen für Klienten und die Verteilung von Leistungen zwischen Klienten unvoreingenommen zu gestalten. Eine Verpflichtung zur Fairness erfordert die Fähigkeit, Unterschiede zwischen Menschen anzuerkennen und sich für Chancen- und Ergebnisgleichheit einzusetzen, sowie eine Diskriminierung von Menschen oder Gruppen aufgrund persönlicher oder sozialer Merkmale zu vermeiden. Mit anderen Worten, Gerechtigkeit bedeutet, dass unfaire Diskriminierung aufgrund bestimmter Merkmale (z.B. Geschlecht oder Alter) nicht stattfindet. Praktiker haben die Pflicht, sich um eine faire Erbringung von Leistungen zu bemühen, diese zugänglich zu machen und den Bedürfnissen potenzieller Klienten entsprechend zu gestalten.

\section{Autonomie}

Autonomie bedeutet, das Recht des Klienten auf Selbstbestimmung zu achten. Dieses Prinzip verdeutlicht die Wichtigkeit, die Fähigkeit eines Klienten zur Eigenverantwortung in Bezug auf die bereitgestellten Leistungen, sowie in alle anderen Lebensbereiche zu entwickeln. Das Prinzip der Autonomie steht der Manipulation von Klienten gegen ihren Willen entgegen, auch wenn diese sozialen Zwecken dient. Praktiker, die die Autonomie ihrer Kunden respektieren:

- gewährleisten, dass jegliche Werbung oder Informationen, die vor der Erbringung von Leistungen angeboten werden, richtig sind;

- holen sich während der gesamten Dauer der professionellen Beziehung die Zustimmung des Klienten ein, welche frei und auf der Basis angemessener Informationen erteilt wird;

- betonen, dass die Teilnahme an den angebotenen Leistungen freiwillig ist;

- treffen explizite Vereinbarungen bevor Leistungen erbracht werden und überprüfen diese Vereinbarungen während der gesamten Dauer der professionellen Beziehung;

- schützen die Privatsphäre ihrer Klienten;

- wahren Vertraulichkeit;

- machen die Weitergabe vertraulicher Informationen in aller Regel von der Zustimmung der betreffenden Person abhängig;

- informieren Klienten vorab über vorhersehbare Interessenskonflikte, spätestens jedoch sobald diese offensichtlich werden. 


\section{Abschnitt Zwei: Anwendung der ethischen Richtlinien in der Praxis}

Dieser Abschnitt enthält mehrere Leitfäden und Beispiele für die kontextabhängige Anwendung der vorliegenden ethischen Richtlinien. Die Beiträge sollen zum Denken anregen und gleichzeitig nützliche Hilfestellungen und einen Leitfaden zur Selbsteinschätzung bieten, um ethische Entscheidungsprozesse in der Praxis zu unterstützen.

Drei wichtige Überlegungen

Vor der Anwendung spezifischer PPI sollten die folgenden drei Aspekte, welche die Grundlage für eine gute Praxis bilden, geprüft und betrachtet werden (Barlow, Allen \& Choate, 2004):

1. Passt die PPI zur vorliegenden Situation? Dies kann durch die Lektüre einschlägiger Literatur und veröffentlichter Erkenntnisse aus zuverlässigen Quellen festgestellt werden. Bevor beispielsweise eine bestimmte PPI zur Förderung von Dankbarkeit angewendet wird, sollte überprüft werden, ob es veröffentlichte Evidenz gibt, die zeigt, dass diese spezifische PPI eine erfolgversprechende Methode für den jeweiligen Klienten ist.

2. Stimmt die PPI mit den aktuellen Bedürfnissen des Klienten überein?

3. Wie wird die Wirksamkeit einer PPI evaluiert? Mit anderen Worten, welches spezifische Ergebnis kann identifiziert und valide gemessen werden, um die Wirksamkeit der PPI zu überprüfen?

\section{Ethische Entscheidungsfindung}

Ethische Entscheidungsfindung kann unterstützt werden durch ausreichende Kenntnis dieser ethischen Richtlinien und der Richtlinien verwandter Berufsstände, das Bewusstmachen tugendhaften und ethischen Verhaltens anderer, sowie den Einsatz persönlicher Stärken bei der Entscheidungsfindung.

Wissen: Wir gehen davon aus, dass die hauptsächlichen Nutzer dieser Richtlinien - nämlich PPP mit entsprechender Ausbildung und Erfahrung in der Ausübung psychologischer oder verwandter Tätigkeiten - mit diesen ethischen Richtlinien und allen ihren Berufsstand betreffenden ethischen Richtlinien vertraut sind. In den meisten Ländern ist gesetzlich vorgeschrieben, dass Praktiker mit einschlägigen Regeln, Normen, Vorschriften, Statuten und Verfahren vertraut sein müssen.

Verhalten: Ethisches Verhalten, insbesondere bei der Durchführung von PPI, kann nicht nur aus Wissen entstehen. Ethisches Verhalten sollte eng mit gesellschaftlichen Vorgängen und Praktiken verbunden sein. Welche Personen in Ihrem beruflichen Umfeld leben ethisches Verhalten vor? Obwohl Ihre Kollegen solche Verhaltensweisen möglicherweise nicht direkt zur Sprache bringen, was an deren Bescheidenheit liegen mag, möchten wir Sie ermutigen, explizit zu fragen, wie Ihre Kollegen und Thre (regionale oder internationale) Fachgemeinschaft ethische Standards einhalten. Das Zusammenstellen eines Fallrepositoriums für gute Praxis wird nützlich sein, um das eigene Verhalten zu reflektieren. Bei einer ausreichenden Anzahl von Fällen werden vermutlich Illustrationen für erstklassige Arbeit zu finden sein. Im Idealfall schaffen Praktiker eine zugängliche Plattform, die auch anderen Praktikern die Möglichkeit bietet, Beiträge zu veröffentlichen.

Stärken einsetzen, um ethische Entscheidungen zu treffen: Stärken können auf verschiedene Weise bei der ethischen Entscheidungsfindung hilfreich sein. Beispielsweise kann die Identifizierung von Stärkenvorbildern bei aktuellen ethischen Entscheidungsfindung helfen. Finden Sie einen 
Kollegen, der als Vorbild für ethisches Verhalten dient. Überlegen Sie, wie er mit einem bestimmten ethischen Dilemma oder einer bestimmten Situation umgegangen ist, oder ganz allgemein, welche spezifischen Stärken, die es wert sind, modelliert zu werden und die bei der Entscheidungsfindung helfen, er im täglichen Umgang mit Kollegen und Klienten verkörpert.

Überlegungen zur beraterischen Arbeit

Es gibt eine Vielzahl von Überlegungen, die bei der Zusammenarbeit mit Klienten zu beachten sind, welche unmittelbare und wichtige ethische Implikationen haben können. Insbesondere sind zu berücksichtigen: Veränderungen bei Klienten, Veränderungen im Umfeld, in dem sich PPP und Klienten befinden, die aktuelle emotionale Verfassung und das Wohlbefinden von Klienten und PPP, die Tendenz der Klienten zur Vermeidung, Machtgefälle in der professionellen Beziehung, übermäßiger Einsatz von Stärken, praktische Weisheit, Nachweis des Ausbildungshintergrunds und der fachlichen Kompetenz, sowie die Würdigung des kulturellen Kontexts. Die Berücksichtigung dieser Aspekte kann helfen, unbeabsichtigte und unerwünschte Folgen abzuschwächen, zu antizipieren oder zu verhindern.

\section{Veränderungen bei Klienten}

Bei der Erbringung psychologischer Dienstleistungen ist es wichtig, das psychologische Wohlbefinden der Klienten während der Behandlung oder Intervention zu beobachten. Bei einige Klienten sinkt das Wohlbefinden trotz der nachgewiesenen Wirksamkeit von PPI. Die Beschwerden können sich aus einer Vielzahl von Gründen verschlimmern, was die Anfälligkeit von Klienten erhöht. Klienten können diese Informationen nicht immer an den Praktiker weitergeben, vielleicht weil sie sich schämen, ängstigen oder befürchten, den Praktiker zu enttäuschen, der an der Steigerung ihres Wohlbefindens arbeitet. Meistens liegt der explizite Fokus von PPI nicht auf der Linderung von Beschwerden, sondern auf der Förderung von Wohlbefinden. Die Beobachtung sich ändernder Umstände im Leben der Kunden ist wichtig, ebenso wie die Anpassung von PPI an diese Veränderungen. Wenn der Kunde beispielsweise eine Trennung erfährt oder seinen Job verliert, ist es wichtig, dass der Praktiker diese Herausforderungen angehen und ihre Auswirkungen bewerten kann. Praktiker sollten in der Lage sein, eine laufende PPI auszusetzen und die Vorgehensweise so anzupassen, dass sie den aktuellen Bedürfnissen von Klienten entspricht oder gegebenenfalls auf eine andere psychosoziale Fachkraft verweisen. Bei Nichtbeachtung kann sich dies nachteilig auf die Allianz zwischen Praktiker und Klient auswirken, was unter Umständen nicht wieder rückgängig zu machen ist.

\section{Veränderungen im Umfeld}

Zusätzlich zur Berücksichtigung von individuellen Veränderungen bei Klienten ist es wichtig, das Umfeld zu berücksichtigen, in dem sowohl Klienten als auch Praktiker eingebunden sind. Nehmen wir einen PPP, der auch Kliniker ist, als Beispiel. Heutzutage arbeiten Kliniker in einem hochkomplexen, schnelllebigen und durch Druck gekennzeichneten Umfeld, das durch Schwierigkeiten wie den Mangel an evidenzbasierten Behandlungsangeboten, mangelnder kultureller Kompetenz, sowie chronischer und komplexer Bedürfnisse von Patienten, die sich auf mehrere Bereiche ihres Lebens auswirken, gekennzeichnet ist. Vor dem Hintergrund dieser Herausforderungen, stellen Sie sich Klienten vor, die:

- ihren Arbeitsplatz oder wichtige Aufträge verloren haben oder für eine verdiente Beförderung nicht berücksichtigt werden, nur weil sie nicht zum Team passen;

- kürzlich mit einer schweren Erkrankung diagnostiziert wurden; 
- mit Betrug oder anderen Formen der Misshandlung durch ihren Partner zu tun haben;

- Diskriminierung erleben;

- zerstörerischen Umwelteinflüssen wie Überschwemmungen oder Waldbränden oder globalen Problemen wie Hungersnöten ausgesetzt sind.

Überlegen Sie, wie sich diese Herausforderungen sich auf Klienten auswirken und die Arbeit mit ihnen beeinflussen können. Diese Fälle werfen eine Vielzahl verschiedener Fragen auf, die auf unterschiedliche Weise betrachtet und angegangen werden müssen, und zwar unter Berücksichtigung der Regularien und Standards des Berufstandes, dem der Praktiker angehört. Daher ist es wichtig, dass PPP, wenn sie PPI anbieten, über ihre Kunden und auch über deren Umfeld Bescheid wissen, sowie über aktuelle Veränderungen der Umstände auf dem Laufenden bleiben. Praktiker müssen sich auch der Herausforderungen in ihrem eigenen Arbeitsumfeld bewusst sein. Dies können administrative Veränderungen sein, die eine Anpassung der Art und Weise der Leistungserbringung erfordern - beispielsweise kann ein neuer Vorgesetzter bestimmte PPI befürworten oder ablehnen oder einen anderen Behandlungsansatz bevorzugen (z.B. auf Achtsamkeit basierende Verfahren, Acceptance Commitment Therapy). Die Änderungen können direkte oder indirekte Auswirkungen auf die Anwendung von PPI haben, auch aus ethischer Perspektive.

Aktuelle emotionale Verfassung und Wohlbefinden

Wenn PPI angeboten werden, ist es wichtig, Veränderungen beim Klienten, sowie deren aktuellen emotionale Verfassung und Wohlbefinden zu messen. Es ist wichtig, dass PPP in den Grenzen ihrer Expertise und Ausbildung praktizieren. Klienten mit psychopathologischen Problemen (z. B. Depressionen, Angstzustände, Traumata) sollten von PPP an eine entsprechend qualifizierte und erfahrene Fachkraft verwiesen werden ${ }^{10}$. Wenn solche Probleme zu Beginn der Arbeitsbeziehungen nicht vorliegen, muss der PPP erkennen, wenn solche Probleme auftreten, während der Kunde eine PPI durchläuft, und sich sofort an eine ausreichend qualifizierte Fachkraft wenden. Im Idealfall bespricht der Praktiker mit dem Klienten, sowie Kollegen und entsprechenden Experten, unter welchen Umständen eine begonnene PPI fortgesetzt werden kann.

\section{Vermeidung}

Vermeidung ist eine der häufigsten Schutzmaßnahmen, die Menschen nutzen, um mit negativen Emotionen umgehen. Ein psychologisch gesundes Leben bedeutet, das gesamte Spektrum an Emotionen bewusst und adaptiv zu nutzen. Einige Klienten, die PPI wünschen, könnten bestrebt sein, tieferliegende negative Emotionen zu vermeiden und PPI in der Hoffnung anwenden, dass sie unangenehme Gefühlszustände zerstreuen oder in Schach halten. Beispiele für Quellen negativer Emotionen sind Erinnerungen an eine bittere Trennung oder Scheidung, Verrat durch einen ansonsten verlässlichen Freund, Groll, Opfer von Diskriminierung geworden zu sein und ungerecht oder voreingenommen von einer Person oder Institution behandelt worden zu sein. Eine Möglichkeit, das Ausmaß von Vermeidung zu beurteilen, besteht darin, auf negative Emotionen zu achten, die wiederholt angesprochen werden, obwohl sie nicht explizit diskutiert werden (d.h., Emotionen, die Kunden nachhaltig belasten und dennoch nicht bewusst thematisiert werden).

\footnotetext{
${ }^{10}$ Anm. d. Ü.: In Deutschland ist dies in der Regel ein Psychologischer Psychotherapeut oder ein Facharzt für Psychiatrie und Psychotherapie.
} 
Obwohl neue Überlegungen und zunehmend belastbare Evidenz zeigen, dass PPI, positive Emotionen und Charakterstärken negative Befindlichkeiten lindern und beheben können (Rashid \& Seligman, 2018), kann dies nur erreicht werden, wenn negative Befindlichkeiten sinnvoll in die Behandlung integriert werden. Wenn starke negative Emotionen von Klienten oder Praktikern vermieden werden, kann die Wirksamkeit der Behandlung beeinträchtigt sein.

Machtgefälle

Eines der wesentlichen Merkmale jeder psychologischen Zusammenarbeit zwischen einem Praktiker und einem Klienten ist die der Beziehung innewohnende Machtdynamik, und insbesondere das Machtgefälle. Therapeuten, Berater, Sozialarbeiter und andere solche Fachleute sind sich dieser Unterschiede in der Regel bewusst. Deshalb sind sie bestrebt, einen sicheren, wertfreien, vertraulichen und einladenden Raum zu schaffen, in dem sich Klienten nicht wie passive Empfänger von verordneten Leistungen fühlen. Nichtsdestotrotz ist die Art der Wechselbeziehung zwischen Praktiker und Klienten so, dass der Praktiker zwangsläufig als Experte wahrgenommen wird. Klienten neigen dazu, zu ihnen aufzuschauen, da sie mit aktuellem Wissen, Training und Erfahrung bei der Erbringung von PPI ausgestattet sind. Daher ist das Machtgefälle immer vorhanden. Der Praktiker muss sich diesem Unterschied bewusst sein und sollte alle Situationen vermeiden, in denen der Klient oder Praktiker eine Entscheidung treffen müssen, die durch das Machtgefälle beeinflusst sein könnte.

\section{Übermäßiger Einsatz von Stärken}

Die unter- oder übertriebene Anwendung einiger stärkebasierter Methoden kann bestehende Schwierigkeiten verschärfen. In verschiedenen Coachingkontexten beispielsweise kann der Vorschlag, dass Kunden vorsichtiger sein sollten, dazu führen, dass einige von ihnen ängstlicher oder unentschlossener werden. Klienten, die vergeben, ohne darüber nachzudenken, wann und wem sie vergeben wollen, können Vergehen übersehen, die, wenn sie nicht geahndet oder verfolgt werden, anderen Schaden zufügen können. Klienten, die übermäßig kreativ sind, können es schwierig finden, Normen oder Regeln einzuhalten, die Gerechtigkeit und Chancengleichheit fördern. Klienten, die sich für faire Entscheidungen an ihrem Arbeitsplatz einsetzen, können Probleme damit bekommen, widersprüchliche Tatsachen in Einklang zu bringen (z.B., die Frage, wie eine gut ausgebildete und gut informierte Person unkluge Entscheidungen treffen kann).

\section{Praktische Weisheit}

Praktiker brauchen, um diese Richtlinien anzuwenden, das, was der Philosoph Aristoteles "phronesis" nannte, d.h. praktische Weisheit. Nehmen wir zum Beispiel einen Praktiker, der mit einer hohen Führungskraft zusammenarbeitet, mit der er eine ausgezeichnete Beziehung aufgebaut hat und die in ihrer Arbeit sehr gute Fortschritte macht. Allerdings stellt der Praktiker fest, dass die Führungskraft in unethisches Verhalten verwickelt ist. Soll der Praktiker die stabile Beziehung erhalten oder die Person konfrontieren und die Beziehung riskieren? Eine einzelne Zusammenstellung von Richtlinien ist möglicherweise nicht ausreichend, um diese Frage zu beantworten. Der Praktiker muss die Situation deuten und widersprüchliche Motive in ein ausgewogenes Verhältnis setzen. In solchen Fällen basiert praktische Weisheit sowohl auf gesammelter Erfahrung als auch auf guten Beziehungen zu Kollegen, die bei ethischen Fragen beraten und helfen können.

\section{Resilient blieben und Burnout verhindern}


Eine Reihe von PP-Konstrukten kann Praktiker vor Burnout schützen. Positive Emotionen (z.B. Hoffnung, Gelassenheit, Freude, Interesse und Ehrfurcht) können die aufmerksamkeits-, kognitions- und verhaltensbezogene Ressourcen von Praktikern aufwerten, die als Puffer gegen Anfälligkeiten wie Burnout-Symptome (z.B. emotionale Erschöpfung) fungieren können. Persönliche Gewohnheiten wie Achtsamkeit können in dieser Hinsicht ebenfalls helfen. Zwischenmenschliche Stärken (z.B. soziale Intelligenz, Freundlichkeit, Liebe, Humor, Lebenssinn und Teamarbeit) können helfen, Herausforderungen wie Depersonalisierung (ein Zustand, in dem die eigenen Gedanken und Gefühle unwirklich oder als nicht zu einem selbst gehörend erscheinen) zu bewältigen. Ebenso kann das Erleben von Lebenssinn und -bedeutung individuelle Gefühle von Depersonalisierung kurieren.

\section{Nachweis des Ausbildungshintergrunds und der fachlichen Kompetenz}

Praktiker sollten die Grenzen ihrer Kompetenz in Bezug auf spezifische psychologische Umstände, spezifische Zielgruppen (z.B., Arbeit mit Kindern, andere Kulturen, Gruppenarbeit) und spezifische PPI darlegen. Beispielsweise kann ein Praktiker Erfahrung in der Beurteilung von Stärken bei Erwachsenen haben, jedoch nicht mit der Beurteilung der Stärken von Kindern oder Jugendlichen. Darüber hinaus kann es passieren, dass der Praktiker sich im Laufe der Erbringung von Leistungen in Bereiche begibt, in denen er nur oberflächlich erfahren oder qualifiziert ist. So kann beispielsweise ein Praktiker zwar erfahren und qualifiziert sein, um Leistungen für Kinder, Jugendliche und Erwachsene zu erbringen, aber nicht ausreichend erfahren oder qualifiziert, diese Leistungen im Rahmen einer Familie anzubieten; in diesem Fall können eine Überweisung oder weitere unterstützende Strukturen erforderlich sein.

Darüber hinaus sollten Praktiker den Ausbildungshintergrund und die einschlägige Erfahrung angeben, auf denen ihre Berufsausübung beruht. Zum Beispiel absolviert ein Praktiker einen sechsmonatigen Zertifikatslehrgang, der eine einwöchige Präsenzphase beinhaltet und ansonsten online absolviert wird. Das Zertifikat wird von einer regionalen oder kommunalen Genehmigungsbehörde nicht anerkannt. Auf der Website des Praktikers steht, dass er ein "Zertifizierter Anwender der Positiven Psychologie" sei. Er hat keine anderen beruflichen Qualifikationen. Ist das ethisch einwandfrei? Es ist wichtig, Thre Kunden ausdrücklich über Art, Umfang und Grenzen Ihrer beruflichen Ausbildung, Praxis und Qualifikation auch über Ihren Berufstitel hinaus zu informieren.

Würdigung des kulturellen Kontextes

Ethische Entscheidungsfindung sollte nicht nur auf Sensibilität, Wissen oder kognitivem Verständnis basieren. Die Entscheidungen sollten auch die besten verfügbaren und relevanten Erkenntnisse und die Umstände des kulturellen Kontextes berücksichtigen. Der kulturelle Kontext ist bei der Durchführung von PPI von entscheidender Bedeutung, denn die Definition dessen, was als normal, gut oder adaptiv begriffen wird, ist von Werten abhängig, die von der Kultur und dem sozialen Kontext, in dem sich das Anliegen oder Dilemma manifestiert, abhängen.

Darüber hinaus unterscheiden sich Kulturen und soziale Normen darin, wie bestimmte Emotionen akzeptiert, ausgedrückt oder verstärkt werden. Daher ist es wichtig, dass die Richtlinien für eine ethische Praxis von PPI auf solchen Erkenntnissen beruhen. Die hier vorgestellten ethischen Richtlinien sind umfassend genug, um dem kulturellen Kontext Rechnung zu tragen, aber gleichzeitig hinreichend detailliert, um individuelle Unterschiede und Bedürfnisse zu berücksichtigen. Die Integration von Erkenntnissen, einschließlich kultureller und individueller Faktoren, kann einfach oder leicht zugänglich sein. Obwohl eine 
solche Integration möglicherweise nicht dazu geeignet ist, Einzelpersonen, die nach ethischer Exzellenz streben, den perfekten Weg, Prozess oder das perfekte Ergebnis vorzugeben, so kann die kollektive Weisheit ihnen dennoch dabei helfen, Fälle, mit denen sie zu tun haben, einzuordnen. So kann nach und nach Exzellenz erreicht werden. Diese Richtlinien sind wichtig, um dem Denken und Handeln von Personen eine Orientierung zu bieten. Diese Richtlinien dienen auch dazu, zu beurteilen, wie stark das eigene Handeln ethischer Exzellenz entspricht. 


\section{Ethische Dilemmata lösen - Eine schrittweise Anleitung}

Obwohl es unmöglich ist, sich vollständig immun gegen ethische Dilemmata zu machen, werden die folgenden Schritte, die weitgehend der Ethics in Psychology (Koocher \& KeithSpiegel, 1998) entnommen sind, zusätzlich zu anderen Informationsquellen, fundierte ethische Entscheidungen in der Praxis erleichtern:

- Praktizieren Sie in Einklang mit allen Gesetzen, Vorschriften und Standards in Bezug auf Ihre Berufsausübung und halten Sie Verhaltensempfehlungen und Richtlinien ein, die von entsprechend autorisierten Einrichtungen oder Behörden in Threr Region festgelegt werden. Diese Informationen helfen Praktikern dabei, ethische Dilemmata zu meistern.

- Erarbeiten Sie sich eine genaue Vorstellung darüber, wie sich das gleiche Ereignis von Einzelfall zu Einzelfall unterschiedlich darstellen kann. Beraten Sie sich mit jemandem, der Ihnen vertraut und der unbefangen ist und klären Sie, ob der vorliegende Sachverhalt ein ethisches Dilemma mit schwerwiegenden Folgen darstellt. Besprechen Sie mit Kollegen, ob das, was Sie wahrnehmen, eine ethische Verfehlung, nur schlechte Berufsetikette oder eine Situation ist, die Einzelnen oder der Öffentlichkeit schaden.

- Nehmen Sie Einsicht in bereits veröffentlichte relevante Richtlinien, Regeln und Standards. Vergewissern Sie sich, dass Sie diese richtig interpretieren und lassen Sie sich bei Bedarf beraten. Schriftliche Richtlinien sind bisweilen abstrakt und unklar, mit viel Raum für eigene Interpretationen.

- Sammeln Sie alle relevanten und hochwertigen Informationen, die Sie finden können. Identifizieren Sie bestehende Wissenslücken und dokumentieren Sie Fakten und Bedenken.

- Beziehen Sie alle diese Informationen in Ihren Überlegungsprozess ein und sprechen Sie mit jemandem, der gut informiert und aufgeschlossen ist und sich nicht scheut, auf die potenziellen Versäumnisse eines Praktikers hinzuweisen.

- Überlegen Sie, wie die sechs ethischen Grundsätze dieser Richtlinien anzuwenden sind (d.h. Wohltätigkeit/Schadensvermeidung, verantwortungsvolle Fürsorge, Achtung der Rechte und Würde des Menschen, Vertrauenswürdigkeit, Gerechtigkeit, Autonomie). Beurteilen Sie, zum Beispiel, welche Rechte, Verantwortlichkeiten und Anfälligkeiten alle Beteiligten haben.

- Erarbeiten Sie mehrere Lösungsansätze und bewerten Sie diese so objektiv wie möglich.

- Überlegen Sie, welche Charakterstärken Ihnen helfen könnten, die beste ethische Entscheidung zu treffen. Könnten Sie sich an Ihren Sinn für Fairness, Ehrlichkeit oder Freundlichkeit wenden? Vielleicht ist Thr Mut nötig, um sich zu motivieren, "das Richtige zu tun"? Denken Sie daran, dass ethische Entscheidungen den klug kombinierten Einsatz mehrerer Charakterstärken erfordern, nicht nur eine Stärke für sich allein oder eine Stärke, die dafür "viel genutzt" wird (Niemiec, 2018).

- Stellen Sie sich das Ergebnis Ihrer Handlung vor und überlegen Sie auch, was passieren könnte, wenn Sie nicht handeln.

- Überlegen Sie, bevor Sie eine Entscheidung treffen, welche Auswirkungen diese Entscheidung auf Sie und alle anderen Beteiligten haben wird und welche systemischen Auswirkungen sich ergeben könnten. Denken Sie auch darüber nach, welche Art von Unterstützung Sie benötigen, um sich an Ihre Entscheidung zu halten.

- Setzen Sie die Entscheidung in konkrete Handlungen um, die dem Geist Threr Entscheidung tatsächlich entsprechen. Sie können Ihre Entscheidung durch eine 
Vielzahl von Handlungen umsetzen. Wählen Sie Handlungen aus, die Ihrer Entscheidung genau und glaubwürdig entsprechen, messbar sind, mit den gewünschten Ergebnissen zusammenhängen und die geringstmögliche Belastung oder Beeinträchtigung für nicht direkt Beteiligte mit sich bringen. 


\section{Anleitung zur Selbsteinschätzung}

Der folgende Abschnitt erläutert Fragen, die Praktiker zum Nachdenken anregen und die dazu beitragen sollen, ethische Dilemmata durch die Verwendung bewährter Verfahren zu vermeiden.

PPI Passung: Entscheiden Sie zuallererst und anhand der besten verfügbaren Evidenz, ob eine bestimmte PPI (z.B. Dankbarkeitstagebuch, Verwendung bestimmter Stärken) zum Klienten, sowie seinen Problemen, Lebensumständen und seiner Behandlungsmotivation passt.

PPI Timing: Welche Schritte werden Sie unternehmen, um zu entscheiden, wann die PPI zweckmäßig ist: 1) zu Beginn der Zusammenarbeit und 2) während die PPI bereits angefangen hat? Unter welchen Umständen würden Sie eine zusätzliche oder alternative Intervention oder Behandlung in Betracht ziehen?

Sensibilität für die eigene psychische Gesundheit: Wie bewusst sind Sie sich Ihres eigenen psychischen Gesundheitszustandes? Die Tatsache, dass Sie psychosoziale Dienstleistungen anbieten, macht Sie nicht immun gegen Symptome psychischer Erkrankungen oder Zuständen, die eine klinische Diagnose erfüllen können. Welche Maßnahmen oder Handlungen ergreifen Sie regelmäßig, um Ihre eigene psychische Gesundheit zu erhalten, um Ihre Klienten bestmöglich betreuen zu können? Welche Hindernisse gibt es bei der Selbstfürsorge, z.B. mangelnde Zeit und Ressourcen, das Gefühl oder der Glaube, dass es Ihnen gut geht oder der Zugang zu qualifizierten Fachleuten, die sich um Ihre psychische Gesundheit kümmern können?

Offenbarungspflicht: Wie wohl fühlen Sie sich dabei, aktiv gegen einen Klienten vorzugehen, der möglicherweise gut auf PPI reagiert, aber Vertraulichkeit wünscht in Bezug auf einen versehentlich berichteten Vorfall (kürzlich oder in der Vergangenheit) oder eine aktuelle Situation, die beispielsweise den Missbrauch von Kindern oder älteren Menschen, sexuellen Missbrauch oder eine unmittelbare Bedrohung für die Sicherheit von Personen betrifft? Wenn Sie nicht nach dem Kodex oder den Richtlinien einer Fachgesellschaft praktizieren, haben Sie eine gesetzliche Anzeigepflicht? ${ }^{11}$

Übertreibung und Generalisierung: Sie treffen auf einer Konferenz jemanden, der von PPI begeistert ist und der Sie zu einem Workshop einlädt, den er anbietet. Sie besuchen den Workshop, der auf einigen wenigen Studien aufbaut, die Ihnen gut vertraut sind. Die Ergebnisse, Anwendungsmöglichkeiten und die Verallgemeinerbarkeit der Ergebnisse der Studien werden übertrieben dargestellt. Die meisten Teilnehmer finden die Präsentation "transformativ" und scheinen die Ergebnisse zu glauben. Was würden Sie tun? Können Sie die Schritte im Abschnitt "Ethische Dilemmata lösen" nutzen, um Ihr Verhalten in einer solchen Situation zu lenken?

\footnotetext{
${ }^{11}$ Anm. d. Ü.: In Deutschland haben psychologische Psychotherapeuten eine Offenbarungs- bzw. Anzeigepflicht, wenn Sie von bevorstehenden Straftaten wir Mord, Totschlag, Geiselnahme, usw. erfahren. Bereits begangene Straftaten unterliegen der Schweigepflicht.
} 


\section{Abschnitt Drei: Fallbeispiele ethischer Praxis}

Die folgenden Fallbeispiele sind Illustrationen von Situationen, die in der Praxis auftreten können und die eine ethische Komponente enthalten, die für die Prinzipien dieser ethischen Richtlinien relevant ist. ${ }^{12}$

\section{Fallbeispiel 1: Kultur - kultursensible Anwendung von PPI}

Ethische Entscheidungen sollten die besten verfügbaren Erkenntnisse gegeben der Umstände des jeweiligen kulturellen Kontextes berücksichtigen. Stellen Sie sich den folgenden Fall vor.

Suzanne ist eine australische Praktikerin, die mit einer Klientin mit ostasiatischem kulturellem Hintergrund arbeitet. Die Klientin wünscht sich eine persönliche Weiterentwicklung und den Fortbestand ihrer familiären Beziehungen. Suzanne entscheidet sich für die Best Possible Self Exercise ${ }^{13}$. Suzanne führt die Klientin durch alle Schritte der Übung. Obwohl die Klientin erhebliche persönliche Fortschritte gemacht hat, berichtet sie, dass die Beziehungen mit ihrer Familie stumpf geworden sind. Aus diesem Grund wird bei wiederholten Online-Messungen keine signifikante Verbesserung des Wohlbefindens der Klientin festgestellt.

Ein wichtiger Aspekt dieses Falls ist die Berücksichtigung des Konzepts des Selbst, das sich von Kultur zu Kultur unterscheidet. Suzanne verfolgt ein Konzept der Entwicklung des Selbst, das oftmals in westlichen Kulturkreisen zu finden ist und das persönliche Weiterentwicklung, sowie neue und weitgehend selbstbestimmte Unternehmungen beinhaltet. Im Gegensatz dazu fokussiert Selbstentwicklung in östlichen (und in den meisten nicht-westlichen) Kulturkreisen stärker auf Investitionen in Beziehungen, die Verbesserung sozialer Interaktionen und den eigenen Beitrag zur Erhaltung von Familien-, Gruppen- und Stammestraditionen. Trotz zunehmender kultureller Vielfalt in den meisten urbanen Metropolen bleiben wesentliche kulturelle Unterschiede oftmals bestehen. Eine wichtige Schlussfolgerung aus diesen Unterschieden ist, dass die Klientin im vorliegenden Fall davon profitieren könnte, zu verstehen, wie sie ihre Charakterstärken nutzen kann, um das Wohl ihrer Familie zu fördern oder familiäre Beziehungen zu vertiefen. Suzanne kann die Charakterstärkeninterventionen Turn Your Strengths Other-Oriented oder Character Strengths Appreciation ${ }^{14}$ (Niemiec, 2018) nutzen, in denen die Klientin lernt, ihre Stärken für den Aufbau von Beziehungen zu nutzen und die eine Möglichkeit bieten, Stärken bei Familienmitgliedern zu erkennen und zu wertschätzen. Es könnte auch sein, dass Selbstentwicklung, die von bedeutenden anderen unterstützt wird, zu einer deutlichen und nachhaltigen Steigerung des Wohlbefindens führt. In einer interdependenten Kultur erfordert Selbstentwicklung, an der wahrscheinlich enge Familienmitglieder beteiligt sind, allerdings auch die Gestaltung komplexerer Wechselwirkungen über einen längeren Zeitraum. Im Gegensatz dazu kann die Best Version of $\mathrm{Me}^{15}$ Intervention, die sich auf die Verbesserung der individuellen Stärken oder das Ergreifen

\footnotetext{
${ }^{12}$ Wir bedanken uns für die Bereitstellung der Fallbeispiele: Fallbeispiele 1 \& 3 - Tayyab Rashid, Fallbeispiel 2 Annalise Roache, Fallbeispiel 4 - Stewart Donaldson, Fallbeispiel 5 - Matthew Iasiello, and Fallbeispiel 6 - Denise Quinlan.

${ }^{13}$ Anm. d. Ü.: Wörtlich übersetzt „Bestmögliches Selbst Übung“، Bei der Intervention werden Klienten gebeten sich ihre bestmögliche Zukunft vorzustellen.

${ }^{14}$ Anm. d. Ü.: Wörtlich übersetzt „Mach deine Stärken auf-andere-ausgerichtet“ und „Wertschätzen von Charakterstärken“"

${ }^{15}$ Anm. d. Ü.: Wörtlich übersetzt „Beste Version meiner Selbst“
} 
einer neuen Initiative konzentriert, eine verhältnismäßig kurze Zeit in Anspruch nehmen. Daher ist es von entscheidender Bedeutung, dass PPP überlegen, wie sie ihre Methoden am sinnvollsten kulturell anpassen können.

Beispielsweise sind die folgenden ethischen Prinzipien für den oben beschriebenen Fall relevant:

- Verantwortungsvolle Fürsorge - Der PPP muss versuchen, die Besonderheiten der Situation des Klienten und dessen kulturellen Hintergrund zu berücksichtigen.

- Wohltätigkeit/Schadensvermeidung - Der PPP obliegt die Pflicht, keinen Schaden anzurichten.

- Achtung der Rechte und Würde des Menschen- Der PPP trägt Verantwortung, sensibel für interkulturelle Gegebenheiten zu sein, sowie die Perspektive und die Bedürfnisse des Klienten zu respektieren

Fallbeispiel 2: Coaching - Coachees in sich verändernden Umständen

Ethische Dilemmata können sich unerwartet stellen und eine Anpassung der angebotenen Leistungen erfordern. Stellen Sie sich den folgenden Fall vor.

Amanda begann mit dem Coaching, weil sie einen Mangel an Orientierung und Sinn im Leben empfand. Früher legte sie viel Wert auf das Vorantreiben ihrer Karriere, jedoch hatte sie inzwischen den Wunsch nach einem ausgeglicheneren Lebensstil entwickelt und war außerdem traurig und enttäuscht, bisher nicht mehr im Leben (außerhalb ihrer beruflichen Entwicklung) erreicht zu haben. In der ersten Sitzung nannte sie mehrere Bereiche, auf die sie sich konzentrieren wollte, darunter Vermögensplanung (um ein Haus zu kaufen), persönliche Entwicklung und die Priorisierung von Freizeit und Geselligkeit. Sie erwähnte, dass ihr aktueller Job herausfordernd sei und dass sie in Zukunft auch eine berufliche Veränderung anstrebe, dies allerdings kein aktuelles Thema für das Coaching sei. In den ersten beiden Sitzungen, die der Formulierung von Zielen und der Klärung von Handlungsschritten dienten, konnten für die Klientin wichtige Fortschritte erzielt werden und das Coaching entwickelte sich insgesamt positiv. Zu Beginn der vierten Sitzung erklärte Amanda jedoch, dass sich ihre Arbeitssituation abrupt verschlechtert habe und sie gekündigt habe, nachdem sie bereits einen neuen Job gefunden hatte. Die Kündigung war nicht gut aufgenommen worden und Amanda fühlte sich verunsichert und emotional an ihrem Arbeitsplatz, wo sie noch vier Wochen verbringen musste, bis ihre Kündigung wirksam würde. Sie erklärte, dass die Reaktion ihres Vorgesetzten auf ihre Kündigung Erinnerungen an ähnliche emotionale Konfrontationen aus ihrer Jugendzeit hervorgerufen hatte.

In dieser Phase war es für den Coach unverzichtbar, seine Fähigkeit zur Selbstregulierung zu nutzen und keinen impulsiven Vorschlag zu machen, jetzt da Amandas Stresslevel anstieg. Stattdessen könnte der Coach die Stärke seiner Neugierde nutzen, um herauszufinden, wie Amanda in naher Zukunft weiterzumachen gedenkt und offen sein für eine Veränderung des thematischen Schwerpunkts der Sitzungen. Es mag auch wichtig sein, die Bedeutung von Amandas emotionaler Reaktion zu verstehen, um zu entscheiden, ob Coaching ein sicherer und geeigneter Ansatz für ihre aktuellen Bedürfnisse ist. Obwohl der Coach mit Amanda eine bestimmte Anzahl von Sitzungen vereinbart haben mag, geben Veränderungen in der Situation des Klienten den richtigen Weg vor. 
Beispielsweise sind die folgenden ethischen Prinzipien für den oben beschriebenen Fall relevant:

- Verantwortungsvolle Fürsorge - Die Hautverantwortung, die dem Coach zufällt, ist, seine Freundlichkeit und Fairness zu nutzen, um das Wohlergehen von Amanda zu schützen und die Grenzen seiner Expertise zu erkennen.

- Autonomie - In diesem Fall könnte sich der Coach darauf konzentrieren, Amandas Fähigkeit zur Selbststeuerung in Bezug auf den Coachingprozess und allen Aspekten ihres Lebens zu entwickeln und die Bedingungen ihrer Zusammenarbeit während des gesamten Prozesses zu überprüfen und anzupassen.

- Wohltätigkeit/Schadensvermeidung - Der Coach, der im besten Interesse von Amanda und auf der Grundlage einer professionellen Einschätzung handelt, sollte sich bewusst sein, dass er strikt innerhalb der Grenzen seiner Kompetenz arbeitet und nur Leistungen erbringt, für die er eine angemessene Ausbildung und einschlägige Erfahrung mitbringt.

\section{Fallbeispiel 3: Klinik - Arbeit mit Traumata}

Viele Menschen, die ein akutes traumatisches Erlebnis haben, sind zunächst einmal überwältigt. Dies kann sich auf viele kulturell unterschiedliche Arten zeigen, insbesondere dann, wenn Klienten gerade in einer PPI involviert sind. Dabei kann es sein, dass Klienten komplexe Strategien entwickeln, um zu vermeiden, an das Trauma zu denken oder sich auf eine Art und Weise zu verhalten, die mit dem Trauma in Zusammenhang steht. Bei der Planung von PPI ist es wichtig, ein solches Trauma (biografisch und/oder aktuell) zu bemerken und richtig einzuschätzen. Der Praktiker sollte, falls er über die nötige Expertise verfügt, intervenieren, ohne das Trauma zu verharmlosen oder zu ignorieren und falls ihm die entsprechende Expertise fehlt, rechtzeitig eine Überweisung vornehmen. Der Praktiker muss auch darauf achten, ob Traumata auftauchen, während der Klient an einer PPI teilnimmt. Der Praktiker muss mit dem Klienten abstimmen und nach Beratung mit Kollegen und anderen Experten entscheiden, wann eine begonnene PPI fortgesetzt werden kann. Stellen Sie sich den folgenden Fall vor.
Salma, 21, ist lange Zeit körperlich, emotional und sexuell missbraucht worden. Sie sucht Hilfe, um mit ihren anhaltenden Gefühlen von Depressionen zurechtzukommen. Sie erwartet, dass der Therapeut explizit nach den traumatischen Ereignissen in ihrem Leben fragen wird. Stattdessen erhält sie einen kurzen Erhebungsbogen, der keine Fragen zu aktuellen oder vergangenen Traumata enthält. In den Beratungsgesprächen selbst stellt Salma fest, dass sie den Therapeuten nicht mag und beschließt, die Einzelheiten ihrer traumatischen Erfahrungen nicht preiszugeben. So bleibt die Behandlung nur oberflächlich wirksam. Nach sechs Monaten bekommt Salma eine neue Therapie verschrieben.

Wie dieser Fall zeigt, ist es wichtig, explizit nach vorhandenen Traumata zu fragen (obwohl dies eine hohe Sensibilität und möglicherweise auch eine klinische Ausbildung erfordert). Abhängig von der Beziehung werden einige Klienten Traumata von selbst ansprechen und nach Ratschlägen, Vorschlägen, Bewältigungsstrategien oder kulturell angemessenen Unterstützungsmöglichkeiten fragen. Es ist wichtig, dass der Praktiker den Bedürfnissen des Klienten entsprechend reagiert, wenn während der Anwendung einer PPI traumatische Erlebnisse aktiviert werden. Zuallererst sollten Praktiker ausreichend qualifiziert, ausgebildet 
und erfahren sein, um mit solchen Situationen umzugehen. Praktiker sollten ihre Stärken von Bescheidenheit und Vorsicht regelmäßig nutzen und stetig überprüfen, ob sie weiterhin Leistungen erbringen sollten, oder umgekehrt, ob der Klient bei jemandem mit spezifischerer Expertise bei der Bereitstellung von Vorschlägen sowie der Entwicklung von Fähigkeiten und Strategien zum Umgang mit Traumata, besser aufgehoben wäre.

Beispielsweise sind die folgenden ethischen Prinzipien für den oben beschriebenen Fall relevant:

- Verantwortungsvolle Fürsorge - Der PPP hat die Verantwortung, Salma in einer Weise zu betreuen, die den Besonderheiten ihrer Situation und ihres Hintergrunds gerecht wird.

- Wohltätigkeit/Schadensvermeidung - Der PPP hat sowohl die Pflicht, Salma keinen weiteren Schaden zuzufügen, als auch ihr Wohlbefinden zu fördern.

\section{Fallbeispiel 4: Im Unternehmenskontext - Druck Daten falsch auszulegen}

Viele positive Arbeits- und Organisationsmaßnahmen werden evaluiert, um ihre Wirksamkeit zu ermitteln. Ethische Dilemmata können unerwartet auftreten, wenn Klienten Befunde nicht akzeptieren und Druck ausüben, bestimmte Ergebnisse verschwinden zu lassen oder falsch darzustellen. Stellen Sie sich den folgenden Fall vor und geben Sie darauf acht, wie eine ganze Reihe von Charakterstärken ungenutzt bleibt, unter anderem Ehrlichkeit/Integrität, Weitblick/Weisheit, Führung und Bescheidenheit.

Die Firma XYZ stellte einen Anwender der PP ein, um die Moral und Leistung der Mitarbeiter zu steigern. In Absprache mit der Unternehmensleitung beschloss der Praktiker, ein Trainingsprogramm zur Erhöhung des psychologischen Kapitals der Mitarbeiter (Hoffnung, Selbstwirksamkeit, Resilienz und Optimismus) durchzuführen.

Die Evaluationsergebnisse zeigen, dass die Mitarbeiter das Training liebten und sich in Zukunft mehr positiv-psychologische Schulungen wünschten. Die Ergebnisse zeigen jedoch auch, dass es keine signifikanten Unterschiede in Bezug auf Hoffnung, Selbstwirksamkeit, Resilienz, Optimismus, Moral oder Leistung zwischen Mitarbeitern, die zufällig für die Teilnahme am Training ausgewählt wurden, und solchen in einer Vergleichsgruppe gab. Die Leiter der Firma XYZ waren so erfreut, dass ihre Mitarbeiter die Schulung genossen, dass sie nicht wollten, dass diese die entmutigenden Evaluationsergebnisse sehen. Stattdessen baten sie den Praktiker zu berichten, dass das Training ein großer Erfolg war und dass in Zukunft mehr Schulungen zu solchen Themen für die Mitarbeiter angeboten würden.

Beispielsweise sind die folgenden ethischen Prinzipien für den oben beschriebenen Fall relevant:

- Autonomie - Hier wird dem Praktizierenden die Handlungsautonomie genommen.

- Wohltätigkeit/Schadensvermeidung - Wohltätigkeit bedeutet eine Verpflichtung zur Förderung des Wohlergehens des Klienten und das von den Führungskräften des Unternehmens befürwortete Maß an Täuschung liegt nicht im besten Interesse der Mitarbeiter. Darüber hinaus erfordert wohltätiges Handeln die Erbringung von Leistungen, die auf einer angemessenen Ausbildung oder entsprechender Berufserfahrung basieren. 
- Vertrauenswürdigkeit - Der Praktiker wird aufgefordert, die Wirksamkeit der PPI zu übertreiben und zu verallgemeinern, was das Vertrauen der Mitarbeiter in den Praktiker missachtet.

\section{Fallbeispiel 5: Mentoring - Motivation "unwilliger" Teilnehmer in einer ungleichen Beziehung}

Oftmals sind diejenigen, die sich einer Intervention am vehementesten widersetzten, diejenigen, die am meisten davon profitieren könnten. PPP sehen sich mit einer kniffligen Situation konfrontiert, wenn sie versuchen, einen Klienten zu motivieren oder zu überzeugen, an einer für ihn vorteilhaften Intervention teilzunehmen, ohne ihn dabei zu manipulieren. Stellen Sie sich folgenden Fall vor.
Abbie, eine Case Managerin in einer Jugendeinrichtung, hat einen Klienten, der sich weigert, an den Übungen einer positiv-psychologischen Gruppenintervention teilzunehmen. Der Leiter der Intervention bat Abbie, ihren Klienten zu motivieren, an den Übungen teilzunehmen und ihn von den Vorteilen der Intervention zu überzeugen. Abbie stimmt mit ihrem Kollegen darin überein, dass die Intervention für den Klienten von großem Nutzen wäre. Abbie ist sich bewusst, dass die Einstellungen und das Verhalten ihres Klienten oft dazu führen, dass er von Maßnahmen ausgeschlossen wird, die wahrscheinlich für ihn hilfreich wären, und beschließt, die Haltung ihres Klienten zur Teilnahme zu hinterfragen. Abbie hat eine vertrauensvolle, unterstützende Beziehung zum Klienten entwickelt und ist nicht bereit, ihre Integrität zu kompromittieren und das Vertrauen des Klienten zu missbrauchen, indem sie die Intervention blind an den Teilnehmer "verkauft" oder Versprechungen macht, die nicht eingehalten werden können. Vielmehr beschreibt sie bescheiden die Belege, die die Intervention stützen, und arbeitet mit ihrem Klienten zusammen, um die Kosten und den Nutzen einer Teilnahme abzuwägen. Sie stellt ihrem Klienten neugierig Fragen über das Für und Wider der Teilnahme an der Intervention und wie die Signaturstärken ihres Klienten im Umfeld der Gruppe genutzt werden könnten. Abbie unterstützt ihren Klienten dabei, Strategien zur Minimierung der Nachteile der Teilnahme zu entwickeln und vor der Teilnahme üben die beiden sowohl eine abgespeckte Variante der Intervention als auch, wie der Klient seine Stärken in der Gruppe einsetzen könnte.

Abbie ist sich sicher, dass ihr Kunde von der Teilnahme an der Intervention profitieren wird und möchte nicht, dass ihr Klient wieder von der Teilnahme ausgeschlossen wird. Ebenso wenig will sie das Vertrauen oder die Autonomie ihres Klienten verraten. Abbie will ihren Klienten herausfordern und ihm helfen, neue Erfahrungen zu machen, befürchtet aber, dass unethische Beeinflussung ihrerseits negative Folgen für ihren Klienten haben und letztlich die Qualität ihrer hart erarbeiteten Beziehung beeinträchtigen könnte. Anstatt die Vorteile der Teilnahme überzubewerten oder diese zu übertreiben, arbeitet Abbie daran, die Einstellung des Kunden zur Intervention zu verstehen, verzerrte Überzeugungen in Frage zu stellen und Möglichkeiten zu schaffen, die Vorteile des Trainings im geschützten Raum der gemeinsamen Sitzungen zu simulieren.

Beispielsweise sind die folgenden ethischen Prinzipien für den oben beschriebenen Fall relevant:

- Gerechtigkeit - Der PPP ist dafür verantwortlich, dass Klienten nicht aufgrund individueller Besonderheiten von Interventionen ausgeschlossen werden. 
- Vertrauenswürdigkeit - Der PPP nutzt die vertrauensvolle Beziehung zum Klienten, um den Klienten zu ermutigen, seine Einstellungen und Überzeugungen zu überprüfen, verrät aber dessen Vertrauen nicht.

- Autonomie - Der PPP respektiert den Willen seines Kunden und ist zögerlich, ihn zu manipulieren.

Fallbeispiel 6: Bildung - Durchführung von Programmen zur Steigerung von Wohlbefinden, wenn bestehende Praktiken dieser Absicht entgegenstehen

Viele Schulen wollen Programme zur Förderung von Wohlbefinden einführen ${ }^{16}$. Sie können dies jedoch aus einer Vielzahl von Gründen tun, inklusive der Sorge um das Wohlbefinden der Schüler, die Bewältigung von Herausforderungen wie Mobbing in der Schule oder der Verbesserung bereits bestehender Förderprogramme. Darüber hinaus kann ein Programm zur Förderung von Wohlbefinden an manchen Schulen auch eine Werbemaßnahme sein, die insbesondere für privaten Schulen ein Alleinstellungsmerkmal sein kann. Stellen Sie sich den folgenden Fall vor.

Ein Lehrer wurde vom Schulleiter gebeten, eine Strategie zur Förderung des Wohlbefindens der Schüler zu entwickeln und einen Plan für die Verabschiedung durch den Schulrat zu erstellen und anschließend eine schnelle Einführung der wichtigsten Bestandteile zu organisieren. Der Schulleiter wollte potenziellen Kunden sagen, dass ihre Kinder von einem speziellen Unterricht zum Thema Wohlbefinden, der im Lehrplan integriert sei, profitieren würden. Den Lehrkräften wurde die Verantwortung für den Glücksunterricht übertragen, obwohl viele sich mit diesem Bereich kaum auskannten. Viele Lehrkräfte waren entsprechend gestresst und fühlten sich auf diesen Unterricht und die damit verbundene zusätzliche Arbeitsbelastung unzureichend vorbereitet. Ein Berater wurde hinzugezogen, um eine halbtägige Weiterbildung zur Verbesserung des Wohlbefindens der Lehrkräfte durchzuführen. Einige Mitarbeiter äußerten sich dem Berater gegenüber besorgt, dass sie ihren Schülern möglicherweise schaden würden, da sie im Bereich der Wohlbefindensförderung schlecht ausgebildet seien und keine Vorkenntnisse darin hätten. Außerdem hatte der Berater gesehen, dass die Schule damit wirbt, Wohlbefinden im Unterricht zu fördern (z.B., wird die Schule als Ort beschrieben, der ein umfassendes Wohlbefinden bei Lehrern und Schülern fördert, und zwar bei jeder Gelegenheit). Weiterhin ist dem Berater bekannt, dass die Schule nach wie vor durch Strafen diszipliniert, die guten Beziehungen zwischen Lehrern und Schülern im Wege steht. Der Lehrer, der für die Umsetzung des Glücksunterrichts verantwortlich gemacht wurde, hat den Berater über diese Problematik informiert. Der Lehrer zögert, die Probleme mit der Schulleitung zu besprechen, nachdem er zuvor von der Gruppe eingeschüchtert wurde, als er ähnliche Bedenken äußerte. Damals wurde seine Loyalität der Schule gegenüber in Frage gestellt und seine Eignung, als Lehrer weiterzuarbeiten, in Frage gestellt.

Beispielsweise sind die folgenden ethischen Prinzipien für den oben beschriebenen Fall relevant:

\footnotetext{
${ }^{16}$ Anm. d. Ü.: Dies stimmt insbesondere für Neuseeland, wo Denise Qinlan, von der das Fallbeispiel stammt, hauptsächlich arbeitet.
} 
- Wohltätigkeit/Schadensvermeidung - Der PPP hat die Pflicht, eine Schule nicht unkritisch als „Wohlbefindensschule“ zu bestätigen, wenn er sich schulischer Praktiken bewusst ist, die das Wohlbefinden der Schüler und Lehrer beeinträchtigen.

- Autonomie - Der Lehrer sollte sich nicht gezwungen fühlen, eine Perspektive oder Position einzunehmen, die im Widerspruch zu seinem eigenen Urteil oder seinen eigenen Werten steht. 


\section{Relevante Richtlinien}

Die folgenden Richtlinien (nach Fachgebieten geordnet und alphabetisch sortiert) wurden als Grundlage für die Entwicklung dieser Richtlinien verwendet:

Psychologie:

- Australia - Psychology - Australian Psychological Society (APS), Code of Ethics (2017).

- Australia - Counselling and Psychotherapy - Psychotherapy \& Counselling Federation of Australia (PACFA), Interim Code of Ethics (2015).

- Canada - Psychology - Canadian Psychology Association (CPA), Canadian Code of Ethics for Psychologists (2017).

- New Zealand - Psychology - New Zealand Psychological Society (NZPS), Code of Ethics for Psychologists (2012, 4th Ed).

- United Kingdom - Counselling and Psychotherapy - British Association for Counselling and Psychotherapy (BACP), Ethical Framework for Good Practice in Counselling and Psychotherapy (2010).

- United States of America - Psychology - American Psychological Association (APA), Ethical Principles of Psychologists and Code of Conduct (2017).

- United States of America - Counselling - American Counselling Association (ACA), Code of Ethics (2014).

Pflege:

- Australia - Nursing - Nursing and Midwifery Board of Australia: Code of Ethics for Nurses (2008).

- New Zealand - Nursing - Nursing Council of New Zealand (NCNZ), Code of Conduct for Nursing (2012).

- United Kingdom - Nursing - Nursing and Midwifery Council (NMC), The Code for Nurses and Midwives (2015).

Coaching:

- International - Coaching - The International Coach Federation (ICF): Core Competencies and Code of Ethics (2015). 


\section{Danksagung}

Die folgenden Personen, die alphabetisch aufgelistet sind, haben einen wesentlichen Beitrag zur Erstellung dieser Richtlinien geleistet, sowie Anregungen und Verbesserungsvorschläge gemacht:

- Associate Professor Aaron Jarden, University of Melbourne, Australia.

- Mr Andrew Alexandra, University of Melbourne, Australia.

- Annalise Roache, Auckland University of Technology, New Zealand.

- Dr Dan Weijers, University of Waikato, Hamilton, New Zealand.

- Dr Denise Quinlan, New Zealand Institute of Wellbeing and Resilience, New Zealand.

- Professor Dianne Vella-Brodrick, University of Melbourne, Australia.

- Giselle Timmerman, Positive Work, United States of America.

- Hein Zegers, European Network of Positive Psychology.

- Dr Helena Agueda Marujo Instituto Superior de Ciências Sociais e Politicas (School of Social and Political Sciences), University of Lisbon, Portugal.

- Professor James Pawelski, University of Pennsylvania, United States of America.

- Dr Jo Mitchell, The Mind Room, Melbourne, Australia.

- Dr Joel Milam, University of Southern California, USA.

- Dr Judy Moskowitz, Northwestern University Feinberg School of Medicine, United States of America.

- Associate Professor Karena Burke, Central Queensland University, Australia.

- Lisa Sansom, LVS Consulting, Canada.

- Margarita Tarragona, Positivamente, Mexico.

- Matthew Iasiello, Wellbeing and Resilience Center, SAHMRI, Australia.

- Dr Mohsen Joshanloo, Keimyung University, South Korea.

- Reb Rebele, University of Melbourne, Australia.

- Dr Rona Hart, former Director MAPP University of East London, England.

- Dr Ryan Niemiec, VIA Institute on Character.

- Scott Asalone, ASGMC Inc, USA.

- Simon Murray, Positive Education Schools Association, Australia.

- Professor Stewart Donaldson, Claremount Graduate University, United States of America.

- Sue Langley, The Langley Group, Australia.

- Dr Suzy Green, The Positivity Institute, Australia.

- Dr Tayyab Rashid, University of Toronto Scarborough, Canada.

- Dr Tim Lomas, University of East London, England. 


\section{Erste Unterzeichner der ethischen Richtlinien für die positiv-psychologische Praxis}

\begin{tabular}{|c|c|c|c|}
\hline $\begin{array}{l}\text { Datum der } \\
\text { ersten } \\
\text { Unterzeichnung }\end{array}$ & Version & $\begin{array}{c}\text { Name und Website der Gesellschaft oder des } \\
\text { Verbandes }\end{array}$ & $\log O$ \\
\hline 19. Juli 2019 & 1.0 & $\begin{array}{l}\text { - VIA Institute on Character } \\
\text { - http://www.viacharacter.org/www/ }\end{array}$ & \\
\hline 19. Juli 2019 & 1.0 & $\begin{array}{l}\text { - Centre for Positive Psychology, } \\
\text { University of Melbourne } \\
\text { - https://education.unimelb.edu.au/cpp }\end{array}$ & \\
\hline 19. Juli 2019 & 1.0 & $\begin{array}{l}\text { - Centre for Positive Psychology, } \\
\text { University of East London } \\
\text { - https://www.uel.ac.uk/postgraduate/msc- } \\
\text { applied-positive-psychology-and- } \\
\text { coaching-psychology }\end{array}$ & \\
\hline 19. Juli 2019 & 1.0 & $\begin{array}{l}\text { - Centre for Positive Psychology, Central } \\
\text { Queensland University } \\
\text { https://www.cqu.edu.au/courses/study- } \\
\text { areas/psychology,-social-work-and- } \\
\text { community- } \\
\text { services/postgraduate/master-of-applied- } \\
\text { positive-psychology }\end{array}$ & \\
\hline 19. Juli 2019 & 1.0 & $\begin{array}{l}\text { - Claremont Evaluation Center, Claremont } \\
\text { Graduate University USA } \\
\text { - https://research.cgu.edu/claremont- } \\
\text { evaluation-center/ }\end{array}$ & \\
\hline 19. Juli 2019 & 1.0 & $\begin{array}{l}\text { - The Wellbeing and Resilience Centre, } \\
\text { Adelaide, Australia } \\
\text { - https://www.wellbeingandresilience.com/ }\end{array}$ & $8 \begin{array}{c}\text { WELLEEING } \\
\text { ANDREESLIENCE } \\
\text { CENTREE }\end{array}$ \\
\hline 19. Juli 2019 & 1.0 & $\begin{array}{l}\text { - The Langley Group } \\
\text { - https://langleygroup.com.au/ }\end{array}$ & Ce Langley Group \\
\hline 19. Juli 2019 & 1.0 & $\begin{array}{l}\text { - New Zealand Institute of Wellbeing and } \\
\text { Resilience } \\
\text { - www.nziwr.co.nz }\end{array}$ & 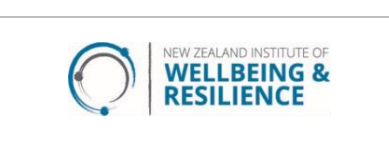 \\
\hline 19. Juli 2019 & 1.0 & $\begin{array}{l}\text { - Middle East Psychological Association, } \\
\text { Division } 1 \text { Positive Psychology }\end{array}$ & 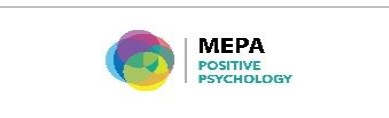 \\
\hline 19. Juli 2019 & 1.0 & $\begin{array}{l}\text { - Humanship } \\
\text { - www.humanshiplb.org }\end{array}$ & $\begin{array}{l}\text { ơf: } \\
\text { HUMANSHIP }\end{array}$ \\
\hline 19. Juli 2019 & 1.0 & - Iranian Positive Psychology Congress & 8 \\
\hline 19. Juli 2019 & 1.0 & $\begin{array}{l}\text { - Japanese Positive Health Psychology } \\
\text { Society } \\
\text { - http://jphp.jp/ }\end{array}$ & 1. 旦本ボジティブサイコロジー医学会 \\
\hline
\end{tabular}




\begin{tabular}{|c|c|c|c|}
\hline 19. Juli 2019 & 1.0 & $\begin{array}{l}\text { - New Zealand Association of Positive } \\
\text { Psychology } \\
\text { - https://www.positivepsychology.org.nz/ }\end{array}$ & $\left(f_{p P}\right.$ \\
\hline 19. Juli 2019 & 1.0 & - Centro de Educacion Emocional Positiva & 83 \\
\hline 19. Juli 2019 & 1.0 & $\begin{array}{l}\text { - National Positive Psychology Association } \\
\text { (NPPA) of India } \\
\text { - http://nppassociation.org/ }\end{array}$ & \\
\hline 19. Juli 2019 & 1.0 & $\begin{array}{l}\text { - Deutsche Gesellschaft für Positive } \\
\text { Psychologie (DGPP) } \\
\text { - https://www.dgpp-online.de/ }\end{array}$ & \\
\hline 19. Juli 2019 & 1.0 & $\begin{array}{l}\text { - Deutschsprachiger Dachverband für } \\
\text { Positive Psychologie (DACH-PP) } \\
\text { - https://www.dach-pp.eu/ }\end{array}$ & D) \\
\hline
\end{tabular}




\section{Autoren}

Aaron Jarden

University of Melbourne, Australia

aaron.jarden@unimelb.edu.au

Tayyab Rashid

University of Toronto Scarborough, Canada

Annalise Roache

Auckland University of Technology, New Zealand

Tim Lomas

University of East London, United Kingdom

\section{Übersetzer}

Johannes Heekerens

Freie Universität Berlin, Deutschland

johannes.heekerens@fu-berlin.de

Aljoscha Dreisörner

Goethe-Universität Frankfurt am Main, Deutschland

dreisoerner@psych.uni-frankfurt.de

\section{Timeline für die Veröffentlichung}

Veröffentlicht am 19. Juli 2019

Deutsche Übersetzung am

\section{Inhaltsverzeichnis}

Barlow, D. H., Allen, L. B., \& Choate, M. L. (2004). Toward a unified treatment for emotional disorders. Behavior Therapy, 35(2), 205-230.

Hone, L., Jarden, A., \& Schofield, G. (2015). An evaluation of positive psychology intervention effectiveness trials using the re-aim framework: A practice-friendly review. Journal of Positive Psychology, 10(4), 303322.

Koocher, G. P. \& Keith-Spiegel, P. (1998). Ethics in psychology: Professional standards and cases. New York, NY: Oxford University Press.

Lomas, T., Hefferon, K., \& Ivtzan, I. (2015). The LIFE model: A meta-theoretical conceptual map for applied positive psychology. Journal of Happiness Studies, 16(5), 1347-1364. https://doi.org/10.1007/s10902-0149563-y

McGrath, R. E. (2014). Character strengths in 75 nations: An update. Journal of Positive Psychology, 10(1), 111. https://doi.org/10.1080/17439760.2014.888580

Niemiec, R. M. (2018). Character strengths interventions: A field-guide for practitioners. Boston: Hogrefe.

Peterson, C., \& Seligman, M. E. P. (2004). Character strengths and virtues: A handbook and classification. New York, NY: Oxford University Press.

Rashid, T. \& Seligman, M. E. P. (2018). Positive psychotherapy: Clinician manual. Oxford, England: Oxford University Press.

Schueller, S. M., Kashdan, T. B., \& Parks, A. C. (2014). Synthesizing positive psychological interventions: Suggestions for conducting and interpreting meta-analyses. International Journal of Wellbeing, 4(1), 9198. https://doi.org/10.5502/ijw.v4i1.5 
Schwartz, S. H. (2006). Basic human values: Theory, measurement, and applications. Jerusalem, Israel: The Hebrew University of Jerusalem.

\section{Literaturhinweise}

Han, H. (2014). Virtue ethics, positive psychology, and a new model of science and engineering ethics education. Science and Engineering Ethics, 21(2), 441-460.

Lomas, T., \& Ivtzan, I. (2016). Professionalising positive psychology: Developing guidelines for training and regulation. International Journal of Wellbeing, 6(3), 96-112.

Koocher, G. P., \& Keith-Spiegel, P. (2008). Ethics in psychology and the mental health professions: Standards and cases (3rd e d.). New York: Oxford University Press.

Sinclair, C. (2017). Ethics in psychology: Recalling the past, acknowledging the present, and looking to the future. Canadian Psychology, 58(1), 20-29.

Vella-Brodrick, D. (2014). Dovetailing ethical practice and positive psychology to promote integrity, industriousness, innovation, and impact. In A. C. Parks \& S. M. Schueller (Eds.), The Wiley Blackwell handbook of positive psychological interventions (pp. 416-432). Chichester, England: Wiley Blackwell. 